\title{
Stochastic evolution equations in Banach spaces and applications to the Heath-Jarrow-Morton-Musiela equations
}

\author{
Zdzisław Brzeźniak ${ }^{1}$ - Tayfun Kok ${ }^{1}$
}

Received: 21 August 2016 / Accepted: 3 July 2018 / Published online: 28 September 2018

(C) The Author(s) 2018

\begin{abstract}
The aim of this paper is threefold. Firstly, we study stochastic evolution equations (with the linear part of the drift being a generator of a $C_{0}$-semigroup) driven by an infinite-dimensional cylindrical Wiener process. In particular, we prove, under some sufficient conditions on the coefficients, the existence and uniqueness of solutions for these stochastic evolution equations in a class of Banach spaces satisfying the so-called $H$-condition. Moreover, we analyse the Markov property of the solutions.

Secondly, we apply the abstract results obtained in the first part to prove the existence and uniqueness of solutions to the Heath-Jarrow-Morton-Musiela (HJMM) equations in weighted Lebesgue and Sobolev spaces.

Finally, we study the ergodic properties of the solutions to the HJMM equations. In particular, we find a sufficient condition for the existence and uniqueness of invariant measures for the Markov semigroup associated to the HJMM equations (when the coefficients are time-independent) in the weighted Lebesgue spaces.

Our paper is a modest contribution to the theory of financial models in which the short rate can be undefined.
\end{abstract}

Keywords Stochastic evolution equations · Heath-Jarrow-Morton-Musiela equations · Markov semigroup · Invariant measures · Martingale-type 2 Banach spaces

Mathematics Subject Classification (2010) 60H15 • 91G80 • 37L40 • 37L55 • 35R10 $\cdot 47 \mathrm{D} 06 \cdot 91 \mathrm{~B} 24 \cdot 91 \mathrm{~B} 25 \cdot 91 \mathrm{~B} 55 \cdot 91 \mathrm{~B} 70$

\section{JEL Classification E43 · C60}

\section{Z. Brzeźniak}

zdzislaw.brzezniak@york.ac.uk

T. Kok

dr.koktayfun@gmail.com

1 Department of Mathematics, University of York, Heslington, York, YO10 5DD, UK 


\section{Introduction}

The theory of stochastic integration in the class of so-called M-type 2 Banach spaces was initiated independently by Neidhardt [44] in 1978 and Dettweiler [23] in 1983. Using this stochastic integration theory, the first of the present authors developed the theory of stochastic evolution equations in M-type 2 Banach spaces; see [5, 6]. In [5], the first author studied linear stochastic evolution equations (with the drift being the infinitesimal generator of an analytic semigroup and the coefficients of the stochastic part being linear operators) driven by a $d$-dimensional Wiener process and proved the existence and uniqueness of solutions for such equations in some real interpolation spaces. In [6], he generalized the results from [5] by proving the existence and uniqueness of solutions for stochastic evolution equations (with the linear part of the drift being the infinitesimal generator of an analytic semigroup and the coefficients satisfying Lipschitz conditions) driven by an infinite-dimensional Wiener process in M-type 2 Banach spaces.

One of the aims of this article is to study stochastic evolution equations driven by an infinite-dimensional cylindrical Wiener process. We continue the line of research originated in [5] and [6]. However, we consider different assumptions on the coefficients. For example, we assume that the linear part of the drift is the infinitesimal generator of a non-analytic $C_{0}$-semigroup. The lack of analyticity of the semigroup leads to some difficulties, and in this article, we show how to overcome this problem. For instance, the time-continuity of the solution cannot be a consequence of the Da Prato-Kwapień-Zabczyk factorisation method, but one needs to use a more sophisticated approach based on the maximal inequality from [14].

The notion of invariant measures is an important topic in the theory of stochastic dynamical systems. Many authors (see, for instance, [24, 25, 22]) have studied the question of the existence and uniqueness of invariant measures for stochastic evolution equations in Hilbert spaces. Recently, the first author, Long and Simão [10] paid attention to the theory of invariant measures for stochastic evolution equations in Banach spaces. They found a sufficient condition about the existence and uniqueness of invariant measures for stochastic evolution equations in Banach spaces, which we introduce and use in this article.

It is now a widely accepted fact that mathematics has a lot of interesting applications in finance. One of these applications appears to be the theory of stochastic evolution equations. The so-called HJMM model proposed by Heath-Jarrow-Morton (HJM) [34] is an example of stochastic evolution equations in finance. This model contains the dynamics of the forward rates. The HJMM model was studied in Hilbert spaces by many authors; see, for instance, [3, 26, 27, 28, 30, 31, 43, 53, 54]. These authors proved the existence and uniqueness of solutions to the HJMM model in some appropriate Hilbert spaces such as the weighted Lebesgue space $L^{2}$ and the weighted Sobolev space $W^{1,2}$. Also some of these authors analysed ergodic properties of the solutions.

Another aim of this article is to apply the abstract results from Sect. 3 to the HJMM model. Thus, we focus on the question of the existence and uniqueness of solutions to the HJMM model in the weighted Lebesgue spaces $\left(L^{p}, p \geq 2\right)$ and the weighted Sobolev spaces $\left(W^{1, p}, p \geq 2\right)$. Also, we aim to study the existence and uniqueness of invariant measures for the HJMM model in the weighted Lebesgue spaces. The 
weighted $L^{p}$ and $W^{1, p}$ spaces, with $p>2$, are 'locally' smaller than the corresponding weighted $L^{2}$ and $W^{1,2}$ spaces. Thus, proving the existence of solutions taking values in these spaces provides additional information about the properties of the solutions. For instance, a solution with values in the weighted $W^{1,2}$ space is locally Hölder-continuous with order strictly less than $1-\frac{1}{2}$, while a solution with values in the weighted $W^{1, p}$ space is locally Hölder-continuous with order strictly less than $1-\frac{1}{p}$. This could have profound consequences in the numerical studies of the HJMM model (to be investigated, for instance, in joint works with Boda Kang from the Department of Mathematics at the University of York). Similar comments apply to the invariant measures. Another motivation is that the use of Sobolev spaces with big $p$ should make it possible to extend the deep results about finite-dimensional realisations, which are usually formulated in terms of the very small set $D\left(A^{\infty}\right)$ to the spaces $W^{1, p}$ or $W^{\theta, p}$ with large $p$ in a similar way to what has been done in [8]. We should point out that as it was proved in [15], the HJMM equations are not well-posed in the space of continuous functions. Hence, one seeks for other type of spaces. So far, the literature has exclusively concentrated on the use of Hilbert spaces (weighted $L^{2}$ or weighted Sobolev $W^{1,2}$ spaces), and our paper is the first attempt to study the HJMM equations in the framework of a specific class of Banach spaces.

Let us now describe briefly the contents of this paper. In Sect. 2, we provide all the necessary preliminaries about the theory of stochastic integration in Banach spaces. In Sect. 3, we study stochastic evolution equations (with the linear part of the drift being only the infinitesimal generator of a $C_{0}$-semigroup and the coefficients satisfying Lipschitz conditions) in the class of Banach spaces satisfying the $H$-condition. Firstly, we prove the existence and uniqueness of solutions for corresponding equations with globally Lipschitz coefficients. Next, using the previous existence result and approximation, we prove the existence and uniqueness of solutions for corresponding equations with locally Lipschitz coefficients. We also analyse the Markov property of the solutions. Finally, we introduce a theorem proposed by Brzeźniak et al. [10] about the existence and uniqueness of invariant measures for corresponding equations with time-independent coefficients. In Sect. 4, we introduce the HeathJarrow-Morton-Musiela (HJMM) equations, but not in detail. Then we apply the abstract results from Sect. 3 to the HJMM equations. In particular, we prove the existence and uniqueness of solutions to the HJMM equations in the weighted Lebesgue and Sobolev spaces, respectively. We also find a sufficient condition for the existence and uniqueness of invariant measures for the Markov semigroup associated to the HJMM equations in the weighted Lebesgue spaces. We mentioned some important features of our results above. Another important feature is that we are able to consider the HJMM equations driven by a cylindrical Wiener process in a (possibly infinite-dimensional) Hilbert space. For this purpose, we use the characterizations of the so-called $\gamma$-radonifying operators from a Hilbert space to an $L^{p}$ space and a Sobolev space $W^{1, p}$ found recently by the first author and Peszat in $[14,13]$.

\section{Stochastic preliminaries}

In this section, we provide all the necessary preliminaries about the theory of stochastic integration in Banach spaces. 
Definition 2.1 A Banach space $\left(X,\|\cdot\|_{X}\right)$ is called martingale-type 2 (or M-type 2) if there exists a constant $C>0$ depending only on $X$ such that for any $X$-valued martingale $\left(M_{n}\right)_{n \in \mathbb{N}}$,

$$
\sup _{n \in \mathbb{N}} \mathbb{E}\left[\left\|M_{n}\right\|_{X}^{2}\right] \leq C \sum_{n} \mathbb{E}\left[\left\|M_{n}-M_{n-1}\right\|_{X}^{2}\right] .
$$

For each $p \geq 2$, the Lebesgue space $L^{p}$ and the Sobolev space $W^{1, p}$ are examples of martingale-type 2 Banach spaces.

Remark 2.2 Another type of Banach space that has been frequently used in the recent years is the UMD, i.e., having the unconditional martingale difference property, Banach space; see $[5,16,46]$. Let us recall that a Banach space $X$ is a UMD space if for any $p \in(1, \infty)$, there exists a constant $\beta_{p}(X)>0$ such that for any $X$-valued martingale $\left(M_{n}\right)_{n \in \mathbb{N}}$ with $M_{0}=0$, for any $\varepsilon: \mathbb{N} \rightarrow\{-1,1\}$ and for any $n \in \mathbb{N}$,

$$
\mathbb{E}\left[\left\|\sum_{j=1}^{n} \varepsilon_{j}\left(M_{j}-M_{j}\right)\right\|_{X}^{p}\right] \leq \beta_{p}(\mathcal{X}) \mathbb{E}\left[\left\|M_{n}\right\|_{X}^{p}\right] .
$$

It is known, see e.g. [51, Proposition 2.4], that if a Banach space $X$ is both UMD and of type 2, then $X$ is of martingale-type 2. From the point of view of applications, in UMD spaces, one can precisely characterise the processes which are stochastically integrable with respect to a cylindrical Wiener process, see [46], while the martingaletype 2 spaces are more suitable for studying general SPDEs; see e.g. [13, 17]. For our purposes, we consider the latter spaces in which all $L^{2}$-integrable processes turn out to be stochastically integrable (see [46, Theorem 3.6]).

It should be pointed out that many important spaces from functional analysis are martingale-type 2 spaces, e.g. classical Sobolev or Besov spaces under certain assumptions on their indices; see e.g. [5, 6]. This has important consequences in numerical analysis and regularity theory. Recently, Hairer and Labbé [32] as well as Liu et al. [40] introduced martingale-type 2 spaces in the theory of regularity structures (and in rough path theory) in order to apply methods from stochastic integration (extending Hölder-type spaces).

Definition 2.3 Let $(\Omega, \mathcal{F}, \mathbb{F}, \mathbb{P})$, where $\mathbb{F}=\left(\mathcal{F}_{t}\right)_{t \geq 0}$, be a filtered probability space. Assume that $H$ is a separable Hilbert space endowed with an inner product $\langle\cdot, \cdot\rangle_{H}$. A family $(W(t))_{t \geq 0}$ of bounded linear operators $W(t): H \rightarrow L^{2}(\Omega, \mathcal{F}, \mathbb{P})$ is called an $H$-valued $\mathbb{F}$-cylindrical canonical Wiener process if

(i) for all $t \geq 0$ and $h_{1}, h_{2} \in H, \mathbb{E}\left[W(t) h_{1} W(t) h_{2}\right]=t\left\langle h_{1}, h_{2}\right\rangle_{H}$;

(ii) for every $h \in H,(W(t) h)_{t \geq 0}$ is a real-valued $\mathbb{F}$-adapted Wiener process.

Definition 2.4 Let $X$ be a separable Banach space and $H$ a separable Hilbert space. A bounded linear operator $L: H \rightarrow X$ is called $\gamma$-radonifying if the image $L\left(\gamma_{H}\right)$ by $L$ of the canonical Gaussian measure $\gamma_{H}$ on $H$ extends to a Gaussian probability measure on $X$. The space of all $\gamma$-radonifying operators from $H$ into $X$ is denoted by $\gamma(H, X)$. 
The following result is originally due to Neidhardt [44, Sect. 4]; see also $[11,38,39,45]$.

Theorem 2.5 Let $X$ be a separable Banach space with a norm $\|\cdot\|_{X}$ and $H$ a separable Hilbert space. Assume that $v_{L}$ is the Gaussian probability measure on $X$ generated by $L \in \gamma(H, X)$. Then the mapping $\|\cdot\|_{\gamma(H, X)}$ defined by

$$
\|L\|_{\gamma(H, X)}=\left(\int_{X}\|x\|_{X}^{2} d \nu_{L}(x)\right)^{\frac{1}{2}}, \quad L \in \gamma(H, X)
$$

is a norm on the space $\gamma(H, X)$ and $\gamma(H, X)$ is a separable Banach space with respect to this norm.

Theorem 2.6 [2, Lemma 8.4] Assume that $H_{1}, H_{2}$ are separable Hilbert spaces and $B_{1}, B_{2}$ are separable Banach spaces. If $h: H_{1} \rightarrow H_{2}$ and $b: B_{2} \rightarrow B_{1}$ are linear bounded operators and $k: H_{2} \rightarrow B_{2}$ is a $\gamma$-radonifying operator, then $k \circ h$ and $b \circ k$ are also $\gamma$-radonifying operators.

The next result is originally due to [44, Lemma 28]; see also [6, Theorem 2.3].

Theorem 2.7 Let $(\Omega, \mathcal{F}, \mathbb{F}, \mathbb{P})$, where $\mathbb{F}=\left(\mathcal{F}_{t}\right)_{t \geq 0}$, be a filtered probability space. Assume that $\left(X,\|\cdot\|_{X}\right)$ is a martingale-type 2 Banach space and $H$ is a separable Hilbert space. Assume that $(W(t))_{t \geq 0}$ is an $H$-valued $\mathbb{F}$-cylindrical canonical Wiener process. If $\xi$ is a $\gamma(H, X)$-valued $\mathbb{F}$-progressively measurable process on $[0, T], T>0$, such that

$$
\mathbb{E}\left[\int_{0}^{T}\|\xi(t)\|_{\gamma(H, X)}^{2} d t\right]<\infty
$$

then the stochastic integral $\int_{0}^{T} \xi(t) d W(t)$ is well defined and there exists a constant $C>0$ such that

$$
\mathbb{E}\left[\left\|\int_{0}^{T} \xi(t) d W(t)\right\|_{X}^{2}\right] \leq C \int_{0}^{T} \mathbb{E}\left[\|\xi(t)\|_{\gamma(H, X)}^{2}\right] d t,
$$

i.e., $\int_{0}^{T} \xi(t) d W(t)$ belongs to the space $L^{2}(\Omega, \mathcal{F}, \mathbb{P} ; X)$.

Theorem 2.8 [6, Theorem 2.4] Suppose that all the assumptions of Theorem 2.7 are satisfied. Let $\xi$ be a $\gamma(H, X)$-valued $\mathbb{F}$-progressively measurable process on $[0, T]$, $T>0$, such that (2.1) holds. Then the process $(\phi(t))_{t \in[0, T]}$ defined by

$$
\phi(t):=\int_{0}^{t} \xi(s) d W(s), \quad t \in[0, T],
$$

is a martingale and has a continuous modification; in particular, it is $\mathbb{F}$-progressively measurable. Moreover, there exists a constant $C>0$ (independent of $\xi$ ) such that

$$
\mathbb{E}\left[\sup _{t \in[0, T]}\|\phi(t)\|_{X}^{2}\right] \leq C \int_{0}^{t} \mathbb{E}\left[\|\xi(s)\|_{\gamma(H, X)}^{2}\right] d s .
$$


Definition 2.9 A Banach space $\left(X,\|\cdot\|_{X}\right)$ is said to satisfy the $H$-condition if for some $q \geq 2$, the function $\psi: X \rightarrow \mathbb{R}$ defined by

$$
\psi(x)=\|x\|_{X}^{q}, \quad x \in X
$$

is of class $C^{2}$ on $X$ (in the Fréchet derivative sense) and if there exist constants $K_{1}, K_{2}>0$ depending on $q$ such that for every $x \in X$,

$$
\left|\psi^{\prime}(x)\right| \leq K_{1}\|x\|_{X}^{q-1} \quad \text { and } \quad\left|\psi^{\prime \prime}(x)\right| \leq K_{2}\|x\|_{X}^{q-2},
$$

where $\psi^{\prime}(x)$ and $\psi^{\prime \prime}(x)$ are the first and second Fréchet derivatives of $\psi$ at $x \in X$.

For each $q \geq p \geq 2$, the Lebesque space $L^{p}$ and the Sobolev space $W^{1, p}$ satisfy the $H$-condition.

Proposition 2.10 [57] If a Banach space $\left(X,\|\cdot\|_{X}\right)$ satisfies the $H$-condition, then it is also a martingale-type 2 Banach space.

Theorem 2.11 [14] Let $(\Omega, \mathcal{F}, \mathbb{F}, \mathbb{P})$, where $\mathbb{F}=\left(\mathcal{F}_{t}\right)_{t \geq 0}$, be a filtered probability space. Assume that $\left(X,\|\cdot\|_{X}\right)$ is a Banach space satisfying the $H$-condition. Assume that $H$ is a separable Hilbert space and $(W(t))_{t \geq 0}$ is an $H$-valued $\mathbb{F}$-cylindrical canonical Wiener process. Let $(S(t))_{t \geq 0}$ be a contraction $C_{0}$-semigroup on $X$. If $\xi$ is a $\gamma(H, X)$-valued $\mathbb{F}$-progressively measurable process on $[0, T], T>0$, such that (2.1) holds, then there exists a constant $K>0$ depending on $H, X$ and $K_{1}, K_{2}$ appearing in the $H$-condition such that

$$
\mathbb{E}\left[\sup _{t \in[0, T]}\left\|\int_{0}^{t} S(t-r) \xi(r) d W(r)\right\|_{X}^{2}\right] \leq K \int_{0}^{T} \mathbb{E}\left[\|\xi(t)\|_{\gamma(H, X)}^{2}\right] d t .
$$

Definition 2.12 Let $\left(X,\|\cdot\|_{X}\right)$ be a Banach space. A $C_{0}$-semigroup $(S(t))_{t \geq 0}$ on $X$ is called contraction-type if there exists a constant $\beta \in \mathbb{R}$ such that

$$
\|S(t)\| \leq e^{\beta t}, \quad t \geq 0
$$

Remark 2.13 If $(S(t))_{t \geq 0}$ is a contraction-type $C_{0}$-semigroup on a Banach space $X$, then the family $(T(t))_{t \geq 0}$ of operators defined by $T(t)=e^{-\beta t} S(t)$ is a contraction $C_{0}$-semigroup on $X$. Therefore, the following corollary follows from Theorem 2.11 .

Corollary 2.14 Let $(\Omega, \mathcal{F}, \mathbb{F}, \mathbb{P})$, where $\mathbb{F}=\left(\mathcal{F}_{t}\right)_{t \geq 0}$, be a filtered probability space. Assume that $\left(X,\|\cdot\|_{X}\right)$ is a Banach space satisfying the $H$-condition. Assume that $H$ is a separable Hilbert space and $(W(t))_{t \geq 0}$ is an $H$-valued $\mathbb{F}$-cylindrical canonical Wiener process. Let $(S(t))_{t \geq 0}$ be a contraction-type $C_{0}$-semigroup on $X$. If $\xi$ is a $\gamma(H, X)$-valued $\mathbb{F}$-progressively measurable process on $[0, T], T>0$, such that $(2.1)$ 
holds, then there exists a constant $K_{T}>0$ depending on $T>0$ and $K$ appearing in (2.2) such that

$$
\mathbb{E}\left[\sup _{t \in[0, T]}\left\|\int_{0}^{t} S(t-r) \xi(r) d W(r)\right\|_{X}^{2}\right] \leq K_{T} \int_{0}^{T} \mathbb{E}\left[\|\xi(t)\|_{\gamma(H, X)}^{2}\right] d t .
$$

\section{Stochastic evolution equations in Banach spaces}

\subsection{Assumptions and definitions}

Throughout this paper, we assume that

- $(\Omega, \mathcal{F}, \mathbb{F}, \mathbb{P})$, where $\mathbb{F}=\left(\mathcal{F}_{t}\right)_{t \geq 0}$, is a filtered probability space.

- $\left(X,\|\cdot\|_{X}\right)$ is a separable Banach space satisfying the $H$-condition.

- $(S(t))_{t \geq 0}$ is a contraction-type $C_{0}$-semigroup on $X$ with infinitesimal generator $A$.

- $\left(H,\langle\cdot, \cdot\rangle_{H}\right)$ is a separable Hilbert space.

- $(W(t))_{t \geq 0}$ is an $H$-valued $\mathbb{F}$-cylindrical canonical Wiener process.

- $F$ and $G$ are maps from $\mathbb{R}_{+} \times X$ into $X$ and $\gamma(H, X)$, respectively (we impose some sufficient conditions on them later).

- $u_{0} \in L^{2}\left(\Omega, \mathcal{F}_{0}, \mathbb{P} ; X\right)$.

We consider the stochastic evolution equation in $X$ given by

$$
\left\{\begin{array}{l}
d u(t)=(A u(t)+F(t, u(t))) d t+G(t, u(t)) d W(t), \quad t>0, \\
u(0)=u_{0}
\end{array}\right.
$$

Definition 3.1 An $X$-valued $\mathbb{F}$-progressively measurable process $(u(t))_{t \geq 0}$ is called a mild solution to (3.1) if for every $t \geq 0$,

$$
\int_{0}^{t} \mathbb{E}\left[\|u(s)\|_{X}^{2}\right] d s<\infty
$$

and $\mathbb{P}$-a.s.

$$
\begin{aligned}
u(t)= & S(t) u_{0}+\int_{0}^{t} S(t-r) F(r, u(r)) d r \\
& +\int_{0}^{t} S(t-r) G(r, u(r)) d W(r), \quad t \geq 0 .
\end{aligned}
$$

\subsection{Existence and uniqueness of solutions to Equation (3.1) with globally Lipschitz coefficients}

Assume that for each $T>0, Z_{T}$ denotes the space of all $X$-valued, continuous, $\mathbb{F}$-adapted processes $u$ on $[0, T]$ such that

$$
\|u\|_{T}:=\left(\mathbb{E}\left[\sup _{t \in[0, T]}\|u(t)\|_{X}^{2}\right]\right)^{\frac{1}{2}}<\infty .
$$

For each $T>0$, the space $Z_{T}$ is a Banach space with respect to the norm $\|\cdot\|_{T}$. 
Lemma 3.2 Assume that $G: \mathbb{R}_{+} \times X \rightarrow \gamma(H, X)$ is a globally Lipschitz map on $X$, i.e., for each $T>0$, there exists a constant $L_{G}>0$ such that for all $t \in[0, T]$,

$$
\left\|G\left(t, x_{1}\right)-G\left(t, x_{2}\right)\right\|_{\gamma(H, X)} \leq L_{G}\left\|x_{1}-x_{2}\right\|_{X}, \quad x_{1}, x_{2} \in X .
$$

Moreover, we assume that for every $x \in X$, the map $G(\cdot, x):[0, \infty) \rightarrow \gamma(H, X)$ is Borel-measurable and for every $T>0$,

$$
\sup _{t \in[0, T]}\|G(t, 0)\|_{\gamma(H, X)}<\infty
$$

Then the mapping $I_{G}: Z_{T} \rightarrow Z_{T}$ defined by

$$
I_{G}(u)(t)=\int_{0}^{t} S(t-r) G(r, u(r)) d W(r), \quad u \in Z_{T}, t \in[0, T]
$$

is well defined. Moreover, it is of linear growth and globally Lipschitz on $Z_{T}$.

Proof Define

$$
M_{T}:=\sup _{t \in[0, T]}\|S(t)\|
$$

Fix $u \in Z_{T}$. We first show that for each $t \geq 0$, the integral $\int_{0}^{t} S(t-r) G(r, u(r)) d W(r)$ is well defined. Fix $t \geq 0$. Due to Theorem 2.6, the process $S(t-r) G(r, u(r))$, $r \in[0, t]$, is $\gamma(H, X)$-valued. Since $G(\cdot, x):[0, \infty) \rightarrow \gamma(H, X)$ is a Borel-measurable function, the process $S(t-r) G(r, u(r)), r \in[0, t]$, is $\mathbb{F}$-progressively measurable. It follows from (3.3) and (3.4) that the map $G$ is of linear growth, i.e., for each $T>0$, there exists a constant $\tilde{L}_{G}>0$ such that for all $t \in[0, T]$,

$$
\|G(t, x)\|_{\gamma(H, X)}^{2} \leq \tilde{L}_{G}^{2}\left(1+\|x\|_{X}^{2}\right), \quad x \in X .
$$

Therefore, by (3.5) and (3.6), we obtain

$$
\mathbb{E}\left[\int_{0}^{t}\|S(t-r) G(r, u(r))\|_{\gamma(H, X)}^{2} d r\right] \leq M_{t}^{2} \tilde{L}_{G}^{2} t+M_{t}^{2} \tilde{L}_{G}^{2} t \mathbb{E}\left[\sup _{r \in[0, t]}\|u(r)\|_{X}^{2}\right] .
$$

Thus, in view of Theorem 2.7, the integral $\int_{0}^{t} S(t-r) G(r, u(r)) d W(r)$ is well defined. Now we show that $I_{G}$ is well defined. By Theorem 2.8, the process $I_{G}(u)$ is continuous and $\mathbb{F}$-adapted. Furthermore, by Corollary 2.14 and (3.6), we get

$$
\mathbb{E}\left[\sup _{t \in[0, T]}\left\|I_{G}(u)(t)\right\|_{X}^{2}\right] \leq T K_{T} \tilde{L}_{G}^{2}+T K_{T} \tilde{L}_{G}^{2} \mathbb{E}\left[\sup _{t \in[0, T]}\|u(t)\|_{X}^{2}\right] .
$$

Hence $I_{G}(u) \in Z_{T}$ and thus $I_{G}$ is well defined. It follows from the last inequality that

$$
\left\|I_{G}(u)\right\|_{T}^{2} \leq T K_{T} \tilde{L}_{G}^{2}\left(1+\|u\|_{T}^{2}\right),
$$


which implies that $I_{G}$ is of linear growth. By Corollary 2.14 and (3.3), we have

$$
\begin{aligned}
\left\|I_{G}\left(u_{1}\right)-I_{G}\left(u_{2}\right)\right\|_{T}^{2} & \leq K_{T} \mathbb{E}\left[\int_{0}^{T}\left\|G\left(t, u_{1}(r)\right)-G\left(t, u_{2}(r)\right)\right\|_{\gamma(H, X)}^{2} d r\right] \\
& \leq K_{T} T L_{G}^{2} \mathbb{E}\left[\sup _{r \in[0, T]}\left\|u_{1}(r)-u_{2}(r)\right\|_{X}^{2}\right], \quad u_{1}, u_{2} \in Z_{T} .
\end{aligned}
$$

Therefore, we infer that

$$
\left\|I_{G}\left(u_{1}\right)-I_{G}\left(u_{2}\right)\right\|_{T} \leq L_{G} \sqrt{K_{T} T}\left\|u_{1}-u_{2}\right\|_{T}, \quad u_{1}, u_{2} \in Z_{T},
$$

which implies that $I_{G}$ is globally Lipschitz on $Z_{T}$.

Lemma 3.3 Assume that $F: \mathbb{R}_{+} \times X \rightarrow X$ is a globally Lipschitz map on $X$, i.e., for each $T>0$, there exists a constant $L_{F}>0$ such that for all $t \in[0, T]$,

$$
\left\|F\left(t, x_{1}\right)-F\left(t, x_{2}\right)\right\|_{X} \leq L_{F}\left\|x_{1}-x_{2}\right\|_{X}, \quad x_{1}, x_{2} \in X .
$$

Moreover, we assume that for every $x \in X$, the function $F(\cdot, x):[0, \infty) \rightarrow X$ is Borel-measurable and for every $T>0$,

$$
\sup _{t \in[0, T]}\|F(t, 0)\|_{X}<\infty
$$

Then the map $I_{F}: Z_{T} \rightarrow Z_{T}$ defined by

$$
I_{F}(u)(t)=\int_{0}^{t} S(t-r) F(r, u(r)) d r, \quad u \in Z_{T}, t \in[0, T],
$$

is well defined. Moreover, it is of linear growth and globally Lipschitz on $Z_{T}$.

Proof Fix $u \in Z_{T}$. By (3.8) and (3.9), $F$ is of linear growth, i.e., for each $T>0$, there exists a constant $\tilde{L}_{F}$ such that for all $t \in[0, T]$,

$$
\|F(t, x)\|_{X}^{2} \leq \tilde{L}_{F}^{2}\left(1+\|x\|_{X}^{2}\right), \quad x \in X .
$$

It follows from (3.5) and (3.10) that the integral $\int_{0}^{t} S(t-r) F(r, u(r)) d r$ is well defined $\mathbb{P}$-a.s. for each $t \geq 0$. Let us now show that $I_{F}$ is well defined. By [37, Proposition 2.22], the process $I_{F}(u)$ is continuous and by [37, Corollary 2.7], it has an $\mathbb{F}$-progressively measurable modification. Moreover, by the Cauchy-Schwarz inequality and (3.5) and (3.10), we obtain

$$
\mathbb{E}\left[\sup _{t \in[0, T]}\left\|I_{F}(u)(t)\right\|_{X}^{2}\right] \leq T^{2} M_{T}^{2} \tilde{L}_{F}^{2}+T^{2} M_{T}^{2} \tilde{L}_{F}^{2} \mathbb{E}\left[\sup _{t \in[0, T]}\|u(r)\|_{X}^{2}\right] .
$$

Therefore $I_{F}(u) \in Z_{T}$ and thus $I_{F}$ is well defined. Moreover, it follows from the last inequality that

$$
\left\|I_{F}(u)\right\|_{T}^{2} \leq T^{2} M_{T}^{2} \tilde{L}_{F}^{2}\left(1+\|u\|_{T}^{2}\right)
$$


which implies that $I_{F}$ is of linear growth. Finally, we show that $I_{F}$ is globally Lipschitz on $Z_{T}$. By the Cauchy-Schwarz inequality and (3.5) and (3.8), we infer that

$$
\begin{aligned}
& \left\|I_{F}\left(u_{1}\right)-I_{F}\left(u_{2}\right)\right\|_{T}^{2} \\
& \leq T \mathbb{E}\left[\sup _{t \in[0, T]} \int_{0}^{t}\left\|S(t-r)\left(F\left(r, u_{1}(r)\right)-F\left(r, u_{2}(r)\right)\right)\right\|_{X}^{2} d r\right] \\
& \leq T M_{T}^{2} L_{F}^{2} \mathbb{E}\left[\sup _{t \in[0, T]} \int_{0}^{t}\left\|u_{1}(r)-u_{2}(r)\right\|_{X}^{2} d r\right] \\
& \leq T^{2} M_{T}^{2} L_{F}^{2} \mathbb{E}\left[\sup _{r \in[0, T]}\left\|u_{1}(r)-u_{2}(r)\right\|_{X}^{2}\right], \quad u_{1}, u_{2} \in Z_{T},
\end{aligned}
$$

which implies that

$$
\left\|I_{F}\left(u_{1}\right)-I_{F}\left(u_{2}\right)\right\|_{T} \leq M_{T} L_{F} T\left\|u_{1}-u_{2}\right\|_{T}, \quad u_{1}, u_{2} \in Z_{T} .
$$

This completes the proof.

Theorem 3.4 Assume that $F: \mathbb{R}_{+} \times X \rightarrow X$ and $G: \mathbb{R}_{+} \times X \rightarrow \gamma(H, X)$ are globally Lipschitz maps on $X$. Moreover, we assume that for every $x \in X$, $F(\cdot, x):[0, \infty) \rightarrow X$ and $G(\cdot, x):[0, \infty) \rightarrow \gamma(H, X)$ are Borel-measurable maps and for every $T>0$,

$$
\sup _{t \in[0, T]}\left(\|F(t, 0)\|_{X}+\|G(t, 0)\|_{\gamma(H, X)}\right)<\infty .
$$

Then there exists a unique $X$-valued continuous mild solution to Eq. (3.1).

Proof By the definition of a mild solution, it is sufficient to show that the integral equation (3.2) has a unique $X$-valued continuous solution. Define the map $\Phi: Z_{T} \rightarrow Z_{T}$ by

$$
\begin{aligned}
\Phi(u)(t)= & S(t) u_{0}+\int_{0}^{t} S(t-r) F(r, u(r)) d r \\
& +\int_{0}^{t} S(t-r) G(r, u(r)) d W(r), \quad u \in Z_{T} .
\end{aligned}
$$

It is obvious that the process $S(\cdot) u_{0}$ is $\mathbb{F}$-adapted. Moreover, for every $\omega \in \Omega$, the map $S(\cdot) u_{0}(\omega):[0, \infty) \rightarrow X$ is continuous and $\mathbb{E}\left[\left\|u_{0}\right\|_{X}^{2}\right]<\infty$. Thus the process $S(\cdot) u_{0}$ belongs to $Z_{T}$. Therefore, by Lemmas 3.2 and 3.3, $\Phi$ is well defined and of linear growth. Moreover, by (3.7) and (3.11), we infer that

$$
\left\|\Phi\left(u_{1}\right)-\Phi\left(u_{2}\right)\right\|_{T} \leq C(T)\left\|u_{1}-u_{2}\right\|_{T}, \quad u_{1}, u_{2} \in Z_{T},
$$

where $C(T)=M_{T} L_{F} T+L_{G} \sqrt{K_{T} T}$. Hence, $\Phi$ is globally Lipschitz on $Z_{T}$. If we choose $T$ small enough, say $T_{0}$, such that $C\left(T_{0}\right) \leq \frac{1}{2}$, then by the Banach fixed point 
theorem, there exists a unique process $u^{1} \in Z_{T_{0}}$ such that $\Phi\left(u^{1}\right)=u^{1}$. Therefore, the integral equation (3.2) has a unique $X$-valued continuous solution $u^{1}$ on $\left[0, T_{0}\right]$.

Define $Z_{(k-1) T_{0}, k T_{0}}, k=1,2,3, \ldots$, to be the space of all $X$-valued, continuous, $\mathbb{F}$-adapted stochastic processes $u$ on $\left[(k-1) T_{0}, k T_{0}\right]$ such that

$$
\mathbb{E}\left[\sup _{t \in\left[(k-1) T_{0}, k T_{0}\right]}\|u(t)\|_{X}^{2}\right]<\infty .
$$

It is obvious that for each $k, Z_{(k-1) T_{0}, k T_{0}}$ is a Banach space endowed with the norm

$$
\|u\|_{(k-1) T_{0}, k T_{0}}=\left(\mathbb{E}\left[\sup _{t \in\left[(k-1) T_{0}, k T_{0}\right]}\|u(t)\|_{X}^{2}\right]\right)^{\frac{1}{2}} .
$$

As above, it can be easily shown that the equation

$$
\begin{aligned}
u(t)= & S\left(t-(k-1) T_{0}\right) u\left((k-1) T_{0}\right)+\int_{(k-1) T_{0}}^{t} S(t-r) F(r, u(r)) d r \\
& +\int_{(k-1) T_{0}}^{t} S(t-r) G(r, u(r)) d W(r)
\end{aligned}
$$

has a unique $X$-valued, continuous solution $u^{k}$ on $\left[(k-1) T_{0}, k T_{0}\right]$ such that

$$
u^{k}\left(k T_{0}\right)=u^{k+1}\left(k T_{0}\right) .
$$

Consequently, we have a sequence $\left(u^{k}\right)_{k \in \mathbb{N}}$ of solutions. Define a process $u$ from these solutions by

$$
u(t)=\sum_{k=1}^{\infty} u^{k}(t) 1_{\left[(k-1) T_{0}, k T_{0}\right]}(t), \quad t \in[0, \infty) .
$$

We claim that this process is a unique $X$-valued continuous solution to the integral equation (3.2). It is obvious that the process $u$ is continuous and $\mathbb{F}$-adapted. Therefore, $u$ is $\mathbb{F}$-progressively measurable. Furthermore, for each $t \geq 0$,

$$
\int_{0}^{t} \mathbb{E}\left[\|u(r)\|_{X}^{2}\right] d r<\infty .
$$

Thus, the integrals in (3.2) are well defined for the process $u$. Let us now show that the process $u$ solves the integral equation (3.2). We have already proved that for $k=1$, the process $u$ on $\left[0, T_{0}\right]$ solves (3.2). By induction, we assume that the process $u$ on $\left[0, k T_{0}\right]$ solves (3.2). Thus, we have

$$
\begin{aligned}
u\left(k T_{0}\right)=u^{k}\left(k T_{0}\right)= & S\left(k T_{0}\right) u_{0}+\int_{0}^{k T_{0}} S\left(k T_{0}-r\right) F(r, u(r)) d r \\
& +\int_{0}^{k T_{0}} S\left(k T_{0}-r\right) G(r, u(r)) d W(r) .
\end{aligned}
$$


We need to show that the process $u$ on $\left[0,(k+1) T_{0}\right]$ solves (3.2). Since $u^{k+1}$ on $\left[k T_{0},(k+1) T_{0}\right]$ solves (3.12), we get, for each $t \in\left[k T_{0},(k+1) T_{0}\right]$,

$$
\begin{aligned}
u^{k+1}(t)= & S\left(t-k T_{0}\right) u^{k+1}\left(k T_{0}\right)+\int_{k T_{0}}^{t} S(t-r) F\left(t, u^{k+1}(r)\right) d r \\
& +\int_{k T_{0}}^{t} S(t-r) G\left(t, u^{k+1}(r)\right) d W(r) .
\end{aligned}
$$

Since $u^{k}\left(k T_{0}\right)=u^{k+1}\left(k T_{0}\right)$ and by (3.13), we obtain

$$
\begin{aligned}
u(t)= & S(t) u_{0}+\int_{0}^{k T_{0}} S(t-r) F(r, u(r)) d r+\int_{0}^{k T_{0}} S(t-r) G(r, u(r)) d W(r) \\
& +\int_{k T_{0}}^{t} S(t-r) F\left(t, u^{k+1}(r)\right) d r+\int_{k T_{0}}^{t} S(t-r) G\left(t, u^{k+1}(r)\right) d W(r) \\
= & S(t) u_{0}+\int_{0}^{t} S(t-r) F(r, u(r)) d r \\
& +\int_{0}^{t} S(t-r) G(r, u(r)) d W(r), \quad t \in\left[0,(k+1) T_{0}\right] .
\end{aligned}
$$

Therefore, the process $u$ on $\left[0,(k+1) T_{0}\right]$ solves (3.2). Hence, the process $u$ is an $X$-valued continuous solution to the integral equation (3.2).

Uniqueness. In principle, the uniqueness of solutions follows from our proof via the Banach fixed point theorem. However, for completeness, we now present our independent proof. Let $u_{1}$ and $u_{2}$ be two solutions to (3.2). Define the process $z$ by $z(t)=u_{1}(t)-u_{2}(t), t \geq 0$. Thus

$$
\begin{aligned}
z(t)= & \int_{0}^{t} S(t-r)\left(F\left(r, u_{1}(r)\right)-F\left(r, u_{2}(r)\right)\right) d r \\
& +\int_{0}^{t} S(t-r)\left(G\left(r, u_{1}(r)\right)-G\left(r, u_{2}(r)\right)\right) d W(r), \quad t \geq 0 .
\end{aligned}
$$

By Theorem 2.8 and (3.3) and (3.5), we have

$$
\begin{aligned}
& \mathbb{E}\left[\left\|\int_{0}^{t} S(t-r)\left(G\left(r, u_{1}(r)\right)-G\left(r, u_{2}(r)\right)\right) d W(r)\right\|_{X}^{2}\right] \\
& \leq C L_{G}^{2} M_{t}^{2} \int_{0}^{t} \mathbb{E}\left[\left\|u_{1}(r)-u_{2}(r)\right\|_{X}^{2}\right] d r .
\end{aligned}
$$

Moreover, using the Cauchy-Schwarz inequality and (3.5) and (3.8), we have

$$
\begin{aligned}
& \mathbb{E}\left[\left\|\int_{0}^{t} S(t-r)\left(F\left(r, u_{1}(r)\right)-F\left(r, u_{2}(r)\right)\right) d r\right\|_{X}^{2}\right] \\
& \leq t L_{F}^{2} M_{t}^{2} \int_{0}^{t} \mathbb{E}\left[\left\|u_{1}(r)-u_{2}(r)\right\|_{X}^{2}\right] d r, \quad t \geq 0 .
\end{aligned}
$$


Taking into account the last two estimates, we infer that

$$
\mathbb{E}\left[\|z(t)\|_{X}^{2}\right] \leq\left(2 t L_{F}^{2} M_{t}^{2}+2 C L_{G}^{2} M_{t}^{2}\right) \int_{0}^{t} \mathbb{E}\left[\|z(r)\|_{X}^{2}\right] d r, \quad t \geq 0 .
$$

Applying Gronwall's lemma, we infer that for all $t \geq 0, \mathbb{E}\left[\|z(t)\|_{X}^{2}\right]=0$. Therefore, for all $t \geq 0, z(t)=0$ and so $u_{1}=u_{2}$. Thus, the process $u$ is a unique $X$-valued continuous solution to the integral equation (3.2). Therefore, the process $u$ is a unique $X$-valued continuous solution to (3.1).

Theorem 3.5 Assume that for each $\zeta \in L^{2}\left(\Omega, \mathcal{F}_{0}, \mathbb{P} ; X\right), u(\cdot, \zeta)$ denotes the solution of (3.1) with the initial value $\zeta$. Then for each $T>0$, there exists a constant $C_{T}>0$ such that for all $t \in[0, T]$,

$$
\mathbb{E}\left[\|u(t, \zeta)\|_{X}^{2}\right] \leq C_{T}\left(1+\mathbb{E}\left[\|\zeta\|_{X}^{2}\right]\right), \quad \zeta \in L^{2}\left(\Omega, \mathcal{F}_{0}, \mathbb{P} ; X\right),
$$

and

$$
\mathbb{E}\left[\|u(t, \zeta)-u(t, \delta)\|_{X}^{2}\right] \leq C_{T} \mathbb{E}\left[\|\zeta-\delta\|_{X}^{2}\right], \quad \zeta, \delta \in L^{2}\left(\Omega, \mathcal{F}_{0}, \mathbb{P} ; X\right)
$$

Proof Fix $T>0$ and $\zeta \in L^{2}\left(\Omega, \mathcal{F}_{s}, \mathbb{P} ; X\right)$. Then for all $t \in[0, T]$,

$$
\begin{aligned}
\mathbb{E}\left[\|u(t, \zeta)\|_{X}^{2}\right] \leq & 3 \mathbb{E}\left[\|S(t) \zeta\|_{X}^{2}\right]+3 \mathbb{E}\left[\left\|\int_{0}^{t} S(t-r) F(r, u(r, \zeta)) d r\right\|_{X}^{2}\right] \\
& +3 \mathbb{E}\left[\left\|\int_{0}^{t} S(t-r) G(r, u(r, \zeta)) d W(r)\right\|_{X}^{2}\right], \quad t \in[0, T] .
\end{aligned}
$$

Using Theorem 2.8 and (3.5) and (3.6), we get, for each $t \in[0, T]$,

$$
\begin{aligned}
& \mathbb{E}\left[\left\|\int_{0}^{t} S(t-r) G(r, u(r, \zeta)) d W(r)\right\|_{X}^{2}\right] \\
& \leq C T \tilde{L}_{G}^{2} M_{T}^{2}+C \tilde{L}_{G}^{2} M_{T}^{2} \int_{0}^{t} \mathbb{E}\left[\|u(r, \zeta)\|_{X}^{2}\right] d r .
\end{aligned}
$$

By the Cauchy-Schwarz inequality and (3.5) and (3.10), we obtain, for each $t \in[0, T]$,

$$
\begin{aligned}
& \mathbb{E}\left[\left\|\int_{0}^{t} S(t-r) F(r, u(r, \zeta)) d r\right\|_{X}^{2}\right] \\
& \leq T^{2} \tilde{L}_{F}^{2} M_{T}^{2}+T \tilde{L}_{F}^{2} M_{T}^{2} \int_{0}^{t} \mathbb{E}\left[\|u(r, \zeta)\|_{X}^{2}\right] d r .
\end{aligned}
$$

Moreover, by (3.5), we have

$$
\mathbb{E}\left[\|S(t) \zeta\|_{X}^{2}\right] \leq M_{T}^{2} \mathbb{E}\left[\|\zeta\|_{X}^{2}\right], \quad t \in[0, T] .
$$


It follows from (3.16)-(3.18) that there exist constants $K_{T}, \tilde{K}_{T}>0$ such that

$$
\mathbb{E}\left[\|u(t, \zeta)\|_{X}^{2}\right] \leq K_{T}+\tilde{K}_{T} \int_{0}^{t} \mathbb{E}\left[\|u(r, \zeta)\|_{X}^{2}\right] d r, \quad t \in[0, T] .
$$

Applying Gronwall's lemma, we obtain the desired result (3.14). Similarly, one can show (3.15).

\subsection{Existence and uniqueness of solutions to Equation (3.1) with locally Lipschitz coefficients}

Lemma 3.6 Assume that $F: \mathbb{R}_{+} \times X \rightarrow X$ is a locally Lipschitz map on balls, i.e., for each $T>0$ and $R>0$, there exists a constant $L_{F}(R)>0$ such that if $t \in[0, T]$ and $\left\|x_{1}\right\|_{X},\left\|x_{2}\right\|_{X} \leq R$, then

$$
\left\|F\left(t, x_{1}\right)-F\left(t, x_{2}\right)\right\|_{X} \leq L_{F}(R)\left\|x_{1}-x_{2}\right\|_{X} .
$$

Moreover, assume that $F$ is of linear growth (uniformly in $t$ ), i.e., for all $T>0$, there exists a constant $\bar{L}_{F}>0$ such that for all $t \in[0, T]$,

$$
\|F(t, x)\|_{X}^{2} \leq \bar{L}_{F}^{2}\left(1+\|x\|_{X}^{2}\right), \quad x \in X .
$$

For each $n \in \mathbb{N}$, define the map $F^{n}: \mathbb{R}_{+} \times X \rightarrow X$ by

$$
F^{n}(t, x)= \begin{cases}F(t, x), & \|x\|_{X} \leq n, t \geq 0 \\ F\left(t, n \frac{x}{\|x\|_{X}}\right), & \|x\|_{X}>n, t \geq 0\end{cases}
$$

Then for each $n \in \mathbb{N}, F^{n}$ is globally Lipschitz on $X$ with constant $3 L_{F}(n)$ (independent of $t$ ). Moreover, $F^{n}$ is of linear growth, i.e.,

$$
\left\|F^{n}(t, x)\right\|_{X}^{2} \leq \bar{L}_{F}^{2}\left(1+\|x\|_{X}^{2}\right), \quad x \in X .
$$

Proof It follows from [7, Lemma 7] that for each $n \in \mathbb{N}, F^{n}$ is globally Lipschitz with constant $3 L_{F}(n)$ (independent of $t$ ). Moreover, it follows from (3.19) that $F^{n}$ is of linear growth.

Similarly, we have the following result.

Lemma 3.7 Assume that $G: \mathbb{R}_{+} \times X \rightarrow \gamma(H, X)$ is a locally Lipschitz map on balls, i.e., for each $T>0$ and $R>0$, there exists a constant $L_{F}(R)>0$ such that if $t \in[0, T]$ and $\left\|x_{1}\right\|_{X},\left\|x_{2}\right\|_{X} \leq R$, then

$$
\left\|G\left(t, x_{1}\right)-G\left(t, x_{2}\right)\right\|_{\gamma(H, X)} \leq L_{G}(R)\left\|x_{1}-x_{2}\right\|_{X} .
$$

Moreover, assume that $G$ is of linear growth (uniformly in $t$ ), i.e., for all $T>0$, there exists a constant $\bar{L}_{G}>0$ such that for all $t \in[0, T]$,

$$
\|G(t, x)\|_{\gamma(H, X)}^{2} \leq \bar{L}_{G}^{2}\left(1+\|x\|_{X}^{2}\right), \quad x \in X .
$$


For each $n \in \mathbb{N}$, define the map $G^{n}: \mathbb{R}_{+} \times X \rightarrow \gamma(H, X)$ by

$$
G^{n}(\cdot, x)= \begin{cases}G(\cdot, x), & \|x\|_{X} \leq n, t \geq 0 \\ G\left(\cdot, n \frac{x}{\|x\|_{X}}\right), & \|x\|_{X}>n, t \geq 0\end{cases}
$$

Then for each $n \in \mathbb{N}, G^{n}$ is globally Lipschitz on $X$ with constant $3 L_{G}(n)$ (independent of $t$ ). Moreover, $G^{n}$ is of linear growth, i.e.,

$$
\left\|G^{n}(t, x)\right\|_{\gamma(H, X)}^{2} \leq \bar{L}_{G}^{2}\left(1+\|x\|_{X}^{2}\right), \quad x \in X .
$$

The previous lemmas yield the following natural conclusion of Theorem 3.4.

Corollary 3.8 Assume the maps $F: \mathbb{R}_{+} \times X \rightarrow X$ and $G: \mathbb{R}_{+} \times X \rightarrow \gamma(H, X)$ are of linear growth and locally Lipschitz on balls. Moreover, we assume that for each $x \in X, F(\cdot, x):[0, \infty) \rightarrow X$ and $G(\cdot, x):[0, \infty) \rightarrow \gamma(H, X)$ are Borel-measurable functions. Then for each $n \in \mathbb{N}$, the stochastic evolution equation

$$
\left\{\begin{array}{l}
d u^{n}(t)=\left(A u^{n}(t)+F^{n}\left(t, u^{n}(t)\right)\right) d t+G^{n}\left(t, u^{n}(t)\right) d W(t), \quad t \geq 0, \\
u^{n}(0)=u_{0}
\end{array}\right.
$$

where $F^{n}$ and $G^{n}$ are the mappings defined in (3.20) and (3.22), respectively, has a unique $X$-valued continuous mild solution.

Lemma 3.9 Assume that for each $n \in \mathbb{N}, u^{n}$ is the unique solution of (3.24). Then for every $T>0$, there exists a constant $C(T)>0$ (independent of $n$ ) such that for each $n \in \mathbb{N}$,

$$
\mathbb{E}\left[\sup _{t \in[0, T]}\left\|u^{n}(t)\right\|_{X}^{2}\right] \leq C(T)
$$

Proof Fix $n \in \mathbb{N}$ and $T>0$. Since $u^{n}$ is the unique solution of (3.24), we have

$$
\begin{aligned}
u^{n}(t)= & S(t) u_{0}+\int_{0}^{t} S(t-r) F^{n}\left(r, u^{n}(r)\right) d r \\
& +\int_{0}^{t} S(t-r) G^{n}\left(r, u^{n}(r)\right) d W(r) \quad t \in[0, T] .
\end{aligned}
$$

Thus, for each $s \in[0, T]$, we obtain

$$
\begin{aligned}
\mathbb{E}\left[\sup _{t \in[0, s]}\left\|u^{n}(t)\right\|_{X}^{2}\right] \leq & 3 \mathbb{E}\left[\sup _{t \in[0, s]}\left\|S(t) u_{0}\right\|_{X}^{2}\right] \\
& +3 \mathbb{E}\left[\sup _{t \in[0, s]}\left\|\int_{0}^{t} S(t-r) F^{n}\left(r, u^{n}(r)\right) d r\right\|_{X}^{2}\right] \\
& +3 \mathbb{E}\left[\sup _{t \in[0, s]}\left\|\int_{0}^{t} S(t-r) G^{n}\left(r, u^{n}(r)\right) d W(r)\right\|_{X}^{2}\right] .
\end{aligned}
$$


By Corollary 2.14 and (3.23), we have, for each $s \in[0, T]$,

$$
\begin{aligned}
& \mathbb{E}\left[\sup _{t \in[0, s]}\left\|\int_{0}^{t} S(t-r) G^{n}\left(r, u^{n}(r)\right) d W(r)\right\|_{X}^{2}\right] \\
& \leq K_{T} \mathbb{E}\left[\int_{0}^{s}\left\|G^{n}\left(r, u^{n}(r)\right)\right\|_{\gamma(H, X)}^{2} d r\right] \\
& \leq K_{T} \bar{L}_{G}^{2} \mathbb{E}\left[\int_{0}^{s}\left(1+\left\|u^{n}(r)\right\|_{X}^{2}\right) d r\right] \\
& \leq K_{T} T \bar{L}_{G}^{2}+K_{T} \bar{L}_{G}^{2} \int_{0}^{s} \mathbb{E}\left[\sup _{t \in[0, r]}\left\|u^{n}(t)\right\|_{X}^{2}\right] d r .
\end{aligned}
$$

Using the Cauchy-Schwarz inequality, (3.5) and (3.21), we obtain

$$
\begin{aligned}
\left\|\int_{0}^{t} S(t-r) F^{n}\left(r, u^{n}(r)\right) d r\right\|_{X}^{2} & \leq t \int_{0}^{t}\left\|S(t-r) F^{n}\left(r, u^{n}(r)\right)\right\|_{X}^{2} d r \\
& \leq t M_{t}^{2} \bar{L}_{F}^{2} \int_{0}^{t}\left(1+\left\|u^{n}(r)\right\|_{X}^{2}\right) d r \\
& \leq t^{2} M_{t}^{2} \bar{L}_{F}^{2}+t M_{t}^{2} \bar{L}_{F}^{2} \int_{0}^{t} \sup _{t \in[0, r]}\left\|u^{n}(t)\right\|_{X}^{2} d r .
\end{aligned}
$$

Therefore, we deduce that

$$
\begin{aligned}
& \mathbb{E}\left[\sup _{t \in[0, s]}\left\|\int_{0}^{t} S(t-r) F^{n}\left(r, u^{n}(r)\right) d r\right\|_{X}^{2}\right] \\
& \leq T^{2} M_{T}^{2} \bar{L}_{F}^{2}+T M_{T}^{2} \bar{L}_{F}^{2} \int_{0}^{s} \mathbb{E}\left[\sup _{t \in[0, r]}\left\|u^{n}(t)\right\|_{X}^{2}\right] d r .
\end{aligned}
$$

Moreover, by (3.5), we get

$$
\mathbb{E}\left[\sup _{t \in[0, s]}\left\|S(t) u_{0}\right\|_{X}^{2}\right] \leq M_{T}\left\|u_{0}\right\|_{X}^{2}, \quad s \in[0, T] .
$$

In view of (3.26)-(3.28), there exist constants $N_{T}>0$ and $V_{T}>0$ such that

$$
\mathbb{E}\left[\sup _{t \in[0, s]}\left\|u^{n}(t)\right\|_{X}^{2}\right] \leq N_{T}+V_{T} \int_{0}^{s} \mathbb{E}\left[\sup _{t \in[0, r]}\left\|u^{n}(t)\right\|_{X}^{2}\right] d r, \quad s \in[0, T] .
$$

Applying Gronwall's lemma, we get the desired result (3.25).

Lemma 3.10 For each $n \in \mathbb{N}$, the random variable $\tau_{n}: \Omega \rightarrow[0, \infty]$ defined by

$$
\tau_{n}(\omega)=\inf \left\{t \in[0, \infty):\left\|u^{n}(t, \omega)\right\|_{X} \geq n\right\}, \quad \omega \in \Omega,
$$

where for each $n \in \mathbb{N}, u^{n}$ is the unique solution of (3.24), is a stopping time. Moreover, the sequence $\left(\tau_{n}\right)_{n \in \mathbb{N}}$ of these stopping times converges to infinity. 
Proof It was proved in [35, Problem 1.2.7] that for each $n \in \mathbb{N}, \tau_{n}$ is a stopping time. Thus we only prove that the sequence $\left(\tau_{n}\right)_{n \in \mathbb{N}}$ converges to infinity. For this aim, we need to show that there exists $\bar{\Omega} \in \mathcal{F}$ with $\mathbb{P}[\bar{\Omega}]=1$ such that for all $\omega \in \bar{\Omega}, \tau_{n}(\omega) \rightarrow \infty$, i.e., for every $T>0$, there exists $k \in \mathbb{N}$ such that for all $n \geq k$, $\tau_{n}(\omega) \geq T$. Therefore, it is sufficient to show that for each $T>0$,

$$
\mathbb{P}\left[\left\{\omega \in \Omega: \exists k \in \mathbb{N} \text { with } \tau_{n}(\omega) \geq T, \forall n \geq k\right\}\right]=1
$$

Fix $T>0$. For each $n \in \mathbb{N}$, set

$$
A_{n}:=\left\{\omega \in \Omega: \sup _{t \in[0, T]}\left\|u^{n}(t)\right\|_{X} \geq n\right\} .
$$

Then by (3.25) and the Chebyshev inequality, we have, for each $n \in \mathbb{N}$,

$$
\mathbb{P}\left[A_{n}\right] \leq C(T) \frac{1}{n^{2}}
$$

Therefore, since $\sum_{n=1}^{\infty} \frac{1}{n^{2}}<\infty$, we have $\sum_{n=1}^{\infty} \mathbb{P}\left[A_{n}\right]<\infty$. Thus, by the BorelCantelli lemma, we infer that $\mathbb{P}\left[\bigcap_{k=1}^{\infty} \bigcup_{n=k}^{\infty} A_{n}\right]=0$ and so

$$
\mathbb{P}\left[\bigcup_{k=1}^{\infty} \bigcap_{n=k}^{\infty}\left(\Omega \backslash A_{n}\right)\right]=1 .
$$

Choose $\bar{\Omega}=\bigcup_{k=1}^{\infty} \bigcap_{n=k}^{\infty}\left(\Omega \backslash A_{n}\right)$ and fix $\omega \in \bar{\Omega}$. Then there is $k \in \mathbb{N}$ such that $\omega \in \bigcap_{n=k}^{\infty}\left(\Omega \backslash A_{n}\right)$, i.e., $\omega \in \Omega \backslash A_{n}, \forall n \geq k$. Therefore, for all $n \geq k$,

$$
\sup _{t \in[0, T]}\left\|u^{n}(t, \omega)\right\|_{X}<n \text {. }
$$

Thus there is $k \in \mathbb{N}$ such that for all $n \geq k$ and $t \in[0, T],\left\|u^{n}(t, \omega)\right\|_{X}<n$, which implies that there is $k \in \mathbb{N}$ such that for all $n \geq k, \tau_{n}(\omega) \geq T$. This gives the desired conclusion.

Theorem 3.11 Assume the maps $F: \mathbb{R}_{+} \times X \rightarrow X$ and $G: \mathbb{R}_{+} \times X \rightarrow \gamma(H, X)$ are of linear growth and locally Lipschitz on balls. Moreover, we assume that for each $x \in X, F(\cdot, x):[0, \infty) \rightarrow X$ and $G(\cdot, x):[0, \infty) \rightarrow \gamma(H, X)$ are Borel-measurable functions. Then there exists a unique $X$-valued continuous mild solution to (3.1).

Proof For each $n \in \mathbb{N}$, let $u^{n}$ be the unique solution of (3.24). Consider the sequence $\left(\tau_{n}\right)_{n \in \mathbb{N}}$ in Lemma 3.10. Define the process $u$ by

$$
u(t)=u^{n}(t), \quad \text { if } t \leq \tau_{n} .
$$

In view of [6, Lemma 4.11], $\left(\tau_{n}\right)_{n \in \mathbb{N}}$ has the two properties that (i) $\tau_{n} \leq \tau_{n+1}$ and (ii) $u^{n}(t, \omega)=u^{n+1}(t, \omega), t \leq \tau_{n}(\omega)$, $\mathbb{P}$-a.s. Therefore, the process $u$ is well defined. We claim that this process is a unique $X$-valued continuous mild solution to (3.1). 
Let us prove this. By the definition of a mild solution, it is sufficient to show that the process $u$ is a unique $X$-valued continuous solution to the integral equation (3.2). It is obvious that $u$ is continuous and $\mathbb{F}$-adapted. Hence, it is $\mathbb{F}$-progressively measurable. Moreover, for each $t \geq 0$,

$$
\int_{0}^{t} \mathbb{E}\left[\|u(r)\|_{X}^{2}\right] d r<\infty
$$

Thus, the integrals in (3.2) are well defined for the process $u$. Now we show that the process $u$ solves (3.2). Since $u^{n}$ is the solution of (3.24), we have the integral equation

$$
\begin{aligned}
u\left(t \wedge \tau_{n}\right)= & u^{n}\left(t \wedge \tau_{n}\right) \\
= & S\left(t \wedge \tau_{n}\right) u_{0}+\int_{0}^{t \wedge \tau_{n}} S\left(t \wedge \tau_{n}-r\right) F^{n}\left(r, u^{n}(r)\right) d r \\
& +\int_{0}^{t \wedge \tau_{n}} S\left(t \wedge \tau_{n}-r\right) G^{n}\left(r, u^{n}(r)\right) d W(r), \quad t \geq 0 .
\end{aligned}
$$

However, we have a problem here. The stochastic integral part of the equation is not well defined because we integrate a process which is not adapted and hence not progressively measurable. To overcome this problem, let us define the process $I$ by

$$
I(t):=\int_{0}^{t} S(t-r) G^{n}\left(r, u^{n}(r)\right) d W(r), \quad t \geq 0 .
$$

It is obvious that $I$ is well defined. Consider the process

$$
I_{\tau_{n}}(t)=\int_{0}^{t} S(t-r) 1_{\left[0, \tau_{n}\right)}(r) G^{n}\left(r \wedge \tau_{n}, u^{n}\left(r \wedge \tau_{n}\right)\right) d W(r), \quad t \geq 0 .
$$

It was shown in [11, Lemma A.1] that if the processes $I$ and $I_{\tau_{n}}$ are continuous, then

$$
S\left(t-t-\tau_{n}\right) I\left(t \wedge \tau_{n}\right)=I_{\tau_{n}}(t) \quad \text { for all } t \geq 0, \mathbb{P} \text {-a.s. }
$$

In particular,

$$
I\left(t \wedge \tau_{n}\right)=I_{\tau_{n}}\left(t \wedge \tau_{n}\right) \quad \text { for all } t \geq 0, \mathbb{P} \text {-a.s. }
$$

Therefore, (3.29) can be rewritten as

$$
\begin{aligned}
u\left(t \wedge \tau_{n}\right)= & S\left(t \wedge \tau_{n}\right) u_{0} \\
& +\int_{0}^{t \wedge \tau_{n}} S\left(t \wedge \tau_{n}-r\right) F^{n}\left(r, u^{n}(r)\right) d r+I_{\tau_{n}}\left(t \wedge \tau_{n}\right), \quad t \geq 0 .
\end{aligned}
$$


Since $r \leq \tau_{n},\left\|u^{n}(r)\right\|_{X} \leq n$. Therefore, from the definitions of $F^{n}$ and $G^{n}$, we get, for every $r \leq \tau_{n}$,

$$
F^{n}\left(r, u^{n}(r)\right)=F\left(r, u^{n}(r)\right) \quad \text { and } \quad G^{n}\left(r, u^{n}(r)\right)=G\left(r, u^{n}(r)\right) .
$$

Also by the definition of $u, u(r)=u^{n}(r)$ if $r \leq \tau_{n}$. Hence, we obtain

$$
\begin{aligned}
u\left(t \wedge \tau_{n}\right)= & S\left(t \wedge \tau_{n}\right) u_{0}+\int_{0}^{t \wedge \tau_{n}} S\left(t \wedge \tau_{n}-r\right) F(r, u(r)) d r \\
& +\int_{0}^{t} S(t-r) 1_{\left[0, \tau_{n}\right)}(r) G\left(r \wedge \tau_{n}, u\left(r \wedge \tau_{n}\right)\right) d W(r) .
\end{aligned}
$$

We know that $\tau_{n} \rightarrow \infty$ and so $t \wedge \tau_{n} \rightarrow t$. Thus $u\left(t \wedge \tau_{n}\right) \rightarrow u(t)$ and $S\left(t \wedge \tau_{n}\right) \rightarrow S(t)$. Therefore, we deduce that

$$
\begin{aligned}
u(t)= & S(t) u_{0}+\int_{0}^{t} S(t-r) F(r, u(r)) d r \\
& +\int_{0}^{t} S(t-r) G(r, u(r)) d W(r), \quad t \geq 0 .
\end{aligned}
$$

Thus, the process $u$ solves the integral equation (3.2). The uniqueness of the solution follows from Theorem 3.4. Thus, the process $u$ is a unique $X$-valued continuous mild solution to (3.1).

\subsection{Markov property and invariant measures}

In this section, we analyse the Markov property of the solution to Eq. (3.1). Because our main interests lies in the existence of an invariant measure, we assume in this and the following subsections that the coefficients in (3.1) are time-independent. It follows from the results proved in the previous sections that (3.1) has a unique $X$-valued continuous mild solution under the Lipschitz assumptions on the coefficients. Similarly, one can show that for every $u_{0} \in L^{2}\left(\Omega, \mathcal{F}_{0}, \mathbb{P} ; X\right)$, Eq. (3.1) with the initial value $u(0)=u_{0}$ has a unique $X$-valued continuous (mild) solution $u$ on $[0, \infty)$. We denote this solution by $u\left(\cdot, u_{0}\right)$. If $u_{0}=x \in X$, we denote the solution by $u(\cdot, x)$. Assume that $B_{b}(X)$ denotes the space of all bounded measurable functions from $X$ into $\mathbb{R}$ and $\mathcal{B}(X)$ the Borel $\sigma$-field of $X$.

Definition 3.12 The family $\left(P_{t}\right)_{t \geq 0}$ of linear bounded operators on $B_{b}(X)$ defined by

$$
\left(P_{t} \varphi\right)(x):=\mathbb{E}[\varphi(u(t, x))], \quad t \geq 0, \varphi \in B_{b}(X), x \in X,
$$

see [50, Definition 1.7], is called the Markov semigroup corresponding to Eq. (3.1).

Remark 3.13 As discussed in [9, Sect. 2.4], for every $\phi \in B_{b}(X)$, the bounded function $P_{t} \phi$ is also measurable when it is well posed, i.e., when weak existence and uniqueness in law hold for Eq. (3.1); see [47, Definition 18 in Corollary 23]. The 
latter generalizes to the infinite-dimensional setting the finite-dimensional result of Stroock and Varadhan [52, Exercise 6.7.4]. Since our results imply weak existence and pathwise uniqueness and since pathwise uniqueness implies uniqueness in law, see [47] for the infinite-dimensional version of the Yamada-Watanabe theory, we infer that this is the case for our problem. Moreover, one can show, see e.g. Ondreját [47], that the family $\left(P_{t}\right)_{t \geq 0}$ is indeed a Markov semigroup, i.e., we have

$$
P_{t+s}=P_{t} P_{s}, \quad t, s \geq 0 .
$$

Definition 3.14 A Borel probability measure $\mu$ on $X$ is called an invariant probability measure for (3.30) if it is an invariant probability measure for the Markov semigroup, i.e., if for any time $t \geq 0$,

$$
\int_{X} P_{t} \phi d \mu=\int_{X} \phi d \mu, \quad \forall \phi \in C_{b}(X),
$$

where $C_{b}(X)$ denotes the space of all bounded and continuous functions from $X$ to $\mathbb{R}$.

Remark 3.15 By Theorem 3.5, the transition semigroup $\left(P_{t}\right)_{t \geq 0}$ is Feller, that is, for any $t \geq 0, P_{t}: C_{b}(X) \rightarrow C_{b}(X)$, where $C_{b}(X)$ is the space of all continuous bounded functions from $X$ into $\mathbb{R}$, is well defined. It is known that the semigroup is in general not strongly continuous on the space $C_{b}(X)$. However, if $\mu$ is an invariant probability measure for the semigroup $\left(P_{t}\right)_{t \geq 0}$, then for every $p \in[1, \infty)$, the semigroup $\left(P_{t}\right)_{t \geq 0}$ has a unique extension to a $C_{0}$-semigroup on the space $L^{p}(X, \mu)$. Such statements can be found in many papers, see e.g. [18] and [42], but we could not find a proof. However, the proof of such a claim is standard since (i) the set of bounded functions in $L^{p}(X, \mu)$ is dense in $L^{p}(X, \mu)$; (ii) for every bounded function $f \in L^{p}(X, \mu)$ and for every $\varepsilon>0$, there exists a continuous bounded function $f_{\varepsilon}: X \rightarrow \mathbb{R}$ such that $\mu\left(\left\{x \in X: f(x) \neq f_{\varepsilon}(x)\right\}\right)<\varepsilon$, see [4, Theorem 7.1.13]; (iii) the trajectories of the process $u(t), t \geq 0$, are continuous. Indeed, properties (i) and (ii) imply that the set $C_{b}(X)$ is dense in $L^{p}(X, \mu)$. See also Da Prato's survey article [19] and Yosida's monograph [56, Theorem XIII.1].

Brzeźniak et al. [10] found some sufficient condition for the existence and uniqueness of an invariant measure for (3.1) with time-independent coefficients. Before introducing this, we present the following natural consequence of Theorem 3.11.

Corollary 3.16 Assume that the maps $F: X \rightarrow X$ and $G: X \rightarrow \gamma(H, X)$ are of linear growth and locally Lipschitz on balls. Then there exists a unique $X$-valued continuous mild solution to the equation

$$
\left\{\begin{array}{l}
d u(t)=(A u(t)+F(u(t))) d t+G(u(t)) d W(t), \quad t \geq 0, \\
u(0)=u_{0} .
\end{array}\right.
$$

Theorem 3.17 [10, Theorem 4.1] Assume that all the assumptions of Corollary 3.16 are satisfied. If there exist constants $\omega>0$ and $n_{0} \in \mathbb{N}$ such that for all $n \geq n_{0}$ and 
$x_{1}, x_{2} \in X$,

$$
\begin{aligned}
& {\left[A_{n}\left(x_{1}-x_{2}\right)+F\left(x_{1}\right)-F\left(x_{2}\right), x_{1}-x_{2}\right]_{X}+\frac{K_{2}(q)}{q}\left\|G\left(x_{1}\right)-G\left(x_{2}\right)\right\|_{\gamma(H, X)}^{2}} \\
& \leq-\omega\left\|x_{1}-x_{2}\right\|_{X}^{2},
\end{aligned}
$$

where $A_{n}$ is the Yosida approximation of $A, K_{2}$ is a constant appearing in the $H$-condition and $[\cdot, \cdot]_{X}$ is the semi-inner product on $X$ (see Definition 3.18 below), then there exists a unique invariant probability measure for (3.30).

Definition 3.18 A semi-inner product on a complex or real vector space $V$ is a mapping $[\cdot, \cdot]_{V}: V \times V \rightarrow \mathbb{C}($ or $\mathbb{R})$ such that

(i) $[x+y, z]_{V}=[x, z]_{V}+[y, z]_{V}, \quad x, y, z \in V$;

(ii) $[\lambda x, y]_{V}=\lambda[x, y]_{V}, \quad x, y \in V, \lambda \in \mathbb{C}$ (or $\mathbb{R}$ );

(iii) $[x, x]_{V}>0 \quad$ for $x \neq 0$;

(iv) $\left|[x, y]_{V}\right|^{2} \leq[x, x]_{V}[y, y]_{V}, \quad x, y \in V$.

Such a vector space $V$ with the semi-inner product $[\cdot, \cdot]_{V}$ is called a semi-inner product space.

Lemma 3.19 [10] The mapping on $X \times X$ defined by

$$
[x, y]_{X}=\left\langle x, y^{*}\right\rangle, \quad x, y \in X,
$$

where $y^{*} \in X^{*}\left(X^{*}\right.$ is the dual space of $\left.X\right)$ is such that we have $\left\|y^{*}\right\|=\|y\|_{X}$ and $\left\langle y, y^{*}\right\rangle=\|y\|_{X}$, is a semi-inner product. Such a $y^{*} \in X^{*}$ exists by the Hahn-Banach theorem.

Remark 3.20 Invariant measures are a subject related to semigroups, and an SDE generates a semigroup only when the coefficients are time-independent. However, there are some papers considering a generalization of an invariant measure for timedependent equations; see e.g. [48, 20, 21]. We plan to investigate this concept in relation to the HJMM equation in the future.

\section{Application to the Heath-Jarrow-Morton-Musiela (HJMM) equations}

\subsection{The HJMM equations}

The value of one dollar at time $t \in[0, T]$ with maturity $T \geq 0$ is called the zerocoupon bond, and is denoted by $P(t, T)$. This is a contract that guarantees the holder one dollar to be paid at the maturity date $T$. Thus, this is the most basic interest rate 
contract. Because of some additional factors like changes of the economy in time, the value of one dollar today could be better than the value of one dollar tomorrow and even the value of one dollar next year. Therefore, the bond prices are unknown in advance. Thus, it is assumed that for each $T \geq 0$ and $t \in[0, T], P(t, T)$ is an $\mathbb{R}$-valued random variable defined on a probability space $(\Omega, \mathcal{F}, \mathbb{P})$. Therefore, for each $T>0,(P(t, T))_{t \in[0, T]}$ is an $\mathbb{R}$-valued stochastic process. Under the assumption that for each $t \in[0, T],[0, \infty) \ni T \mapsto P(t, T)$ is a differentiable function, the function $f$ defined by

$$
f(t, T)=-\frac{\partial}{\partial T} \log P(t, T), \quad T>0, t \in[0, T],
$$

is called the forward rate function. It contains all the original bond price information. For each $T>0$, the family $(f(t, T))_{t \in[0, T]}$ is a stochastic process, and called forward rate process. For each $t \in[0, T]$, the function $[t, \infty) \ni T \mapsto f(t, T)$ is called the forward curve. It is always assumed that the forward curves are locally integrable with respect to Lebesgue measure. If in addition $P(T, T)=1$, then one can write the equality

$$
P(t, T)=e^{-\int_{t}^{T} f(t, s) d s}, \quad T>0, t \in[0, T] .
$$

In the framework of Heath-Jarrow-Morton [34], it was assumed that for each $T>0$, the forward rate process $(f(t, T))_{t \in[0, T]}$ satisfies for $t \in[0, T]$ the stochastic differential equation

$$
d f(t, T)=\left(\sum_{i}^{d} \sigma_{i}(t, T) \int_{t}^{T} \sigma_{i}(t, u) d u\right) d t+\sum_{i}^{d} \sigma_{i}(t, T) d W_{i}(t),
$$

where $W(t)=\left(W_{1}(t), W_{2}(t), \ldots, W_{d}(t)\right), t \geq 0$, is a standard $d$-dimensional Brownian motion and for each $T>0,\left(\sigma_{i}(t, T)\right)_{t \in[0, T]}$ is a real-valued stochastic process. Filipović [26] extended the framework of Heath-Jarrow-Morton by considering a Wiener process in a (possibly infinite-dimensional) Hilbert space instead of a standard finite-dimensional Brownian motion. Thus, he assumed that for an arbitrary but fixed $T>0$, the forward rate process $(f(t, T))_{t \in[0, T]}$ satisfies the stochastic differential equation

$$
d f(t, T)=\left\langle\sigma(t, T), \int_{t}^{T} \sigma(t, u) d u\right\rangle_{H} d t+\langle\sigma(t, T), d W(t)\rangle_{H}, \quad t \in[0, T],
$$

where $(W(t))_{t \geq 0}$ is a Wiener process in an infinite-dimensional Hilbert space $H$ endowed with an inner product $\langle\cdot, \cdot\rangle_{H}$ and for each $T>0,(\sigma(t, T))_{t \in[0, T]}$ is an $H$-valued stochastic process.

Using the Musiela parametrization [43], an important connection between the HJMM model and stochastic partial differential equations can be provided as follows. Define

$$
r(t)(x)=f(t, t+x), \quad T=t+x, \quad x, t \geq 0,
$$


where $x$ is called time to maturity, and for each $t \geq 0$, the function $r(t)$ is a random variable on $(\Omega, \mathcal{F}, \mathbb{P})$ taking values in the space of real-valued functions on $[0, \infty)$ and called forward curve. Therefore, the family $(r(t))_{t \geq 0}$ of forward curves is a stochastic process taking values in the space of real-valued functions on $[0, \infty)$ and called forward curve process. By the framework of Heath-Jarrow-Morton [34], the forward curve process $(r(t))_{t \geq 0}$ satisfies the stochastic partial differential equation

$$
\begin{aligned}
d r(t)(x)= & \left(\frac{\partial}{\partial x} r(t)(x)+\left\langle\alpha(t)(x), \int_{0}^{x} \alpha(t)(y) d y\right\rangle_{H}\right) d t \\
& +\langle\alpha(t)(x), d W(t)\rangle_{H}, \quad t \geq 0,
\end{aligned}
$$

where $\alpha$ is the function defined by

$$
\alpha(t)(x)=\sigma(t, t+x), \quad t \geq 0, x \in[0, \infty) .
$$

Let $g:[0, \infty) \times[0, \infty) \times \mathbb{R} \rightarrow H$ be a given function which is locally integrable with respect to the second variable. Assume that the volatility $\alpha$ is defined by

$$
\alpha(t)(x)=g(t, x, r(t)(x)), \quad t, x \geq 0,
$$

i.e., the volatility $\alpha$ depends on the forward curve process $(r(t))_{t \geq 0}$. Then (4.2) becomes

$$
\begin{aligned}
d r(t)(x)= & \left(\frac{\partial}{\partial x} r(t)(x)+\left\langle g(t, x, r(t)(x)), \int_{0}^{x} g(t, y, r(t)(y)) d y\right\rangle_{H}\right) d t \\
& +\langle g(t, x, r(t)(x)), d W(t)\rangle_{H} .
\end{aligned}
$$

This equation is known as the Heath-Jarrow-Morton-Musiela (HJMM) equation. In this paper, we analyse, under some sufficient conditions on the function $g$, the existence and uniqueness of solutions to (4.3) in certain Banach spaces. Moreover, we analyse the existence and uniqueness of invariant measures for (4.3).

\subsection{Existence and uniqueness of solutions to the HJMM equations in weighted Lebesgue spaces}

In this section, we study the existence and uniqueness of solutions to the HJMM equations in the weighted $L^{p}$ spaces with $p \geq 2$. The motivation for this is at least threefold. Firstly, it is of purely theoretical curiosity to see to what extent the results obtained in the $L^{2}$-framework remain valid in the more general one. The second one, to which our paper is the very first step, is to consider the HJMM equations in the weighted fractional Sobolev spaces $W^{\theta, p}$ with $\theta>\frac{1}{p}$ (allowing thus to have $\theta<1$ ). Taking $p$ large and $\theta$ just a bit bigger than $\frac{1}{p}$ will allow us to use a space which in 
some sense is much closer to the space $C$ of continuous functions. Also using either weighted $L^{p}$ or fractional Sobolev spaces $W^{\theta, p}$ allows one to consider the HJMM equations with coefficients satisfying less stringent regularity assumptions than in the classical approach. Thirdly, if the functions $\sigma_{i}(t, \cdot)$ are not regular enough, then the natural state spaces for the solutions of the HJMM equations are the weighted $L^{p}$ spaces; see Example 4.10. Let us point out that in their monograph [50], Peszat and Zabczyk used the weighted $L^{2}$ spaces.

For each $v \in \mathbb{R}$ and $p \geq 1$, define $L_{v}^{p}$ to be the space of all (equivalence classes of) Lebesgue-measurable functions $f:[0, \infty) \rightarrow \mathbb{R}$ such that

$$
\|f\|_{\nu, p}:=\left(\int_{0}^{\infty}|f(x)|^{p} e^{\nu x} d x\right)^{\frac{1}{p}}<\infty .
$$

For each $v \in \mathbb{R}$ and $p \geq 1, L_{v}^{p}$ is a separable Banach space with the norm $\|\cdot\|_{\nu, p}$.

Lemma 4.1 For each $v>0$ and $p \geq 1$, the space $L_{v}^{p}$ is continuously embedded into the space $L^{1}$. In particular,

$$
\|f\|_{1} \leq\left(\frac{p}{v q}\right)^{\frac{1}{q}}\|f\|_{v, p}, \quad f \in L_{v}^{p},
$$

where $q \in[1, \infty)$ is such that $\frac{1}{p}+\frac{1}{q}=1$.

The proof follows from the Hölder inequality.

Theorem 4.2 Let $v>0$ and $p \geq 2$. Assume that $g:[0, \infty) \times[0, \infty) \times \mathbb{R} \rightarrow H$ is a measurable function with respect to the second variable such that there exist functions $\bar{g} \in L_{v}^{p}$ and $\hat{g} \in L_{v}^{p} \cap L^{\infty}$ such that for all $t \in[0, \infty)$,

$$
\|g(t, x, u)\|_{H} \leq|\bar{g}(x)|, \quad u \in \mathbb{R}, x \in[0, \infty),
$$

and

$$
\|g(t, x, u)-g(t, x, v)\|_{H} \leq|\hat{g}(x) \| u-v|, \quad u, v \in \mathbb{R}, x \in[0, \infty) .
$$

Then for each $r_{0} \in L^{2}\left(\Omega, \mathcal{F}_{0}, \mathbb{P} ; L_{v}^{p}\right)$, (4.3) with the function $g$ has a unique $L_{v}^{p}$-valued continuous mild solution with the initial value $r(0)=r_{0}$.

The proof is given below.

Lemma 4.3 For each $v \in \mathbb{R}$ and $p \geq 2$, the space $L_{v}^{p}$ satisfies the $H$-condition. In particular, if $\psi$ is the function defined by

$$
\psi: L_{v}^{p} \ni f \mapsto \psi(f)=\|f\|_{\nu, p}^{p} \in \mathbb{R},
$$


then

$$
\left\|\psi^{\prime}(f)\right\| \leq p\|f\|_{\nu, p}^{p-1} \quad \text { and } \quad\left\|\psi^{\prime \prime}(f)\right\| \leq p(p-1)\|f\|_{\nu, p}^{p-2}, \quad f \in L_{v}^{p},
$$

where $\psi^{\prime}(f)$ and $\psi^{\prime \prime}(f)$ are the first and second Fréchet derivatives of $\psi$ at $f \in L_{v}^{p}$, respectively.

Proof Fix $v \in \mathbb{R}$ and $p \geq 2$. Define the linear operator $T: L_{v}^{p} \rightarrow L^{p}$ by

$$
T f(x)=f(x) e^{\frac{v}{p} x}, \quad f \in L_{v}^{p}, x \in[0, \infty) .
$$

It is obvious that the map $T$ is well defined, bijective and

$$
\|T f\|_{p}=\|f\|_{\nu, p}, \quad f \in L_{v}^{p} .
$$

It is clear that any linear operator, so $T$, is twice Fréchet differentiable and for each $f \in L_{v}^{p}, T^{\prime}(f)=T$ and $T^{\prime \prime}(f)=0$. By [13, Proposition 2.1], for every $p \geq 2$, the space $L^{p}$ satisfies the $H$-condition for any $q \geq p$, i.e., for some $q \geq p$ (in particular, $q=p)$, the map $\phi: L^{p} \ni f \mapsto \phi(f)=\|f\|_{p}^{p} \in \mathbb{R}$ is of class $C^{2}$ and

$$
\left\|\phi^{\prime}(f)\right\| \leq p\|f\|_{p}^{p-1} \quad \text { and } \quad\left\|\phi^{\prime \prime}(f)\right\| \leq p(p-1)\|f\|_{p}^{p-2}, \quad f \in L^{p} .
$$

We can write $\psi=\phi \circ T$. Since $T$ and $\phi$ are of class $C^{2}, \psi$ is of class $C^{2}$. Moreover, it follows from (4.6) and (4.7) that

$$
\left\|\psi^{\prime}(f)\right\| \leq p\|f\|_{\nu, p}^{p-1}, \quad f \in L_{v}^{p} .
$$

Similarly,

$$
\left\|\psi^{\prime \prime}(f)\right\| \leq p(p-1)\|f\|_{\nu, p}^{p-2}, \quad f \in L_{v}^{p},
$$

which finishes the proof.

Lemma 4.4 For each $v>0$ and $p \geq 1$, the shift semigroup $(S(t))_{t \geq 0}$ on the space $L_{v}^{p}$ is a contraction-type $C_{0}$-semigroup, in particular,

$$
\|S(t)\| \leq e^{\frac{-v t}{p}}, \quad t \geq 0 .
$$

Moreover, its infinitesimal generator $A$ is characterized by

$$
\mathcal{D}(A)=\left\{f \in L_{v}^{p}: D f \in L_{v}^{p}\right\}
$$

and

$$
A f=D f, \quad f \in \mathcal{D}(A),
$$

where $D$ is the weak derivative (of order one). 
This result can be proved along the lines of [56, Examples IX.2.1 and IX.5.1]. See also [37, Lemma 5.3] for the first and [37, Lemma 5.8] for the second part.

For each $v \in \mathbb{R}$ and $p \geq 1$, define $L_{v}^{p}(H)$ to be the space of all (equivalence classes of) Borel-measurable functions $f:[0, \infty) \rightarrow H$ such that

$$
\|f\|_{L_{v}^{p}(H)}:=\left(\int_{0}^{\infty}\|f(x)\|_{H}^{p} e^{v x} d x\right)^{\frac{1}{p}}<\infty .
$$

For each $v \in \mathbb{R}$ and $p \geq 1, L_{v}^{p}(H)$ is a separable Banach space endowed with the norm $\|\cdot\|_{L_{v}^{p}(H)}$. The following lemma gives a sufficient condition under which an $L_{v}^{p}$-valued operator defined on $H$ is $\gamma$-radonifying.

Lemma 4.5 For each $v \in \mathbb{R}$ and $p \geq 2$, the bounded linear operator $K: H \rightarrow L_{v}^{p}$ defined by

$$
K[h](x)=\langle\kappa(x), h\rangle_{H}, \quad h \in H, x \in[0, \infty),
$$

where $\kappa \in L_{v}^{p}(H)$, is a $\gamma$-radonifying operator, i.e., $K \in \gamma\left(H, L_{v}^{p}\right)$. Moreover, there exists a constant $N>0$ independent of $\kappa$ such that

$$
\|K\|_{\gamma\left(H, L_{v}^{p}\right)} \leq N\|\kappa\|_{L_{v}^{p}(H)} .
$$

Proof Define the linear operator $V: L_{v}^{p}(H) \rightarrow L^{p}(H)$ by

$$
V f=f e^{\frac{v}{p}}, \quad f \in L_{v}^{p}(H) .
$$

It is obvious that $V$ is well defined, bijective and

$$
\|V f\|_{L^{p}(H)}=\|f\|_{L_{v}^{p}(H)}, \quad f \in L_{v}^{p}(H) .
$$

Fix $\kappa \in L_{v}^{p}(H)$ so that $V \kappa \in L^{p}(H)$. By [12, Proposition 2.1], the bounded linear operator $M: H \rightarrow L^{p}$ defined by

$$
M[h](x)=\langle\phi(x), h\rangle_{H}, \quad h \in H, x \in[0, \infty),
$$

where $\phi \in L^{p}(H)$, is a $\gamma$-radonifying operator and, for a constant $N>0$ independent of $\phi$,

$$
\|M\|_{\gamma\left(H, L^{p}\right)} \leq N\|\phi\|_{L^{p}(H)} .
$$

Therefore, the operator $\bar{K}: H \rightarrow L^{p}$ defined by

$$
\bar{K}[h](x)=\langle V \kappa(x), h\rangle_{H}, \quad h \in H, x \in[0, \infty),
$$

is a $\gamma$-radonifying operator, and for a constant $N>0$,

$$
\|\bar{K}\|_{\gamma\left(H, L^{p}\right)} \leq N\|V \kappa\|_{L^{p}(H)} .
$$


It follows from (4.8) that

$$
\|\bar{K}\|_{\gamma\left(H, L^{p}\right)} \leq N\|\kappa\|_{L_{v}^{p}(H)} .
$$

For the linear operator $T$ in the proof of Lemma 4.3, we can write $K=T^{-1} \circ \bar{K}$. Thus by Theorem 2.6, the map $K$ is a $\gamma$-radonifying operator. Moreover, since $\left\|T^{-1}\right\| \leq 1$ and by (4.9), we infer that

$$
\|K\|_{\gamma\left(H, L_{v}^{p}\right)} \leq\left\|T^{-1}\right\|\|\bar{K}\|_{\gamma\left(H, L^{p}\right)} \leq N\|\kappa\|_{L_{v}^{p}(H)},
$$

which completes the proof.

Lemma 4.6 Let $v>0$ and $p \geq 2$. Assume $g:[0, \infty) \times[0, \infty) \times \mathbb{R} \rightarrow H$ is a function satisfying the assumptions of Theorem 4.2. Then $F:[0, \infty) \times L_{v}^{p} \rightarrow L_{v}^{p}$ defined by

$$
F(t, f)(x)=\left\langle g(t, x, f(x)), \int_{0}^{x} g(t, y, f(y)) d y\right\rangle_{H}, \quad f \in L_{\nu}^{p}, x, t \in[0, \infty),
$$

is well defined. Moreover, we have:

(i) For all $t \in[0, \infty)$ and $f \in L_{\nu}^{p}$,

$$
\|F(t, f)\|_{\nu, p} \leq\left(\frac{p}{v q}\right)^{\frac{1}{q}}\|\bar{g}\|_{\nu, p}^{2}
$$

(ii) $F$ is globally Lipschitz on $L_{v}^{p}$ with a Lipschitz constant independent of time $t$.

Proof Fix $t \geq 0$ and $f \in L_{v}^{p}$. It follows from (4.4) that for each $x \in[0, \infty)$, the integral $\int_{0}^{x} g(t, y, f(y)) d y$ exists. Define the function $h$ by

$$
h(x)=\int_{0}^{x} g(t, y, f(y)) d y, \quad x \in[0, \infty) .
$$

This function is continuous. Indeed, by (4.4), we have for every sequence $\left(x_{n}\right)_{n \in \mathbb{N}}$ in $[0, \infty)$ converging to $x \in[0, \infty)$ that as $n \rightarrow \infty$,

$$
\left|h\left(x_{n}\right)-h(x)\right| \leq \int_{x}^{x_{n}}|g(t, y, f(y))| d y \leq \int_{0}^{\infty} 1_{\left[x, x_{n}\right]}(y)|\bar{g}(y)| d y \longrightarrow 0 .
$$

Thus $h$ is continuous and so it is measurable. Therefore, $F(t, f)$ is measurable. Moreover, by the Cauchy-Schwarz inequality, Lemma 4.1 and (4.4), we obtain

$$
\begin{aligned}
|F(t, f)(x)| & \leq \| g\left(t, x, f(x)\left\|_{H}\right\| \int_{0}^{x} g(t, y, f(y)) d y \|_{H}\right. \\
& \leq|\bar{g}(x)| \int_{0}^{\infty}|\bar{g}(y)| d y \leq\left(\frac{p}{v q}\right)^{\frac{1}{q}}\|\bar{g}\|_{v, p}|\bar{g}(x)|, \quad x \in[0, \infty) .
\end{aligned}
$$


Taking into account the last inequality, we deduce that

$$
\int_{0}^{\infty}|F(t, f)(x)|^{p} e^{v x} d x \leq\left(\frac{p}{v q}\right)^{\frac{p}{q}}\|\bar{g}\|_{\nu, p}^{2 p} .
$$

Therefore $F(t, f) \in L_{v}^{p}$ and thus $F$ is well defined. Moreover, (4.11) gives the desired conclusion (4.10). Finally, we prove that $F$ is globally Lipschitz on $L_{v}^{p}$. Fix $t \geq 0$ and $f_{1}, f_{2} \in L_{v}^{p}$. Then by the Cauchy-Schwarz inequality, we have

$$
\begin{aligned}
& \left|F\left(t, f_{1}\right)(x)-F\left(t, f_{2}\right)(x)\right| \\
& \leq\left|\left\langle g\left(t, x, f_{1}(x)\right), \int_{0}^{x}\left(g\left(t, y, f_{1}(y)\right)-g\left(t, y, f_{2}(y)\right)\right) d y\right\rangle_{H}\right| \\
& \quad+\left|\left\langle g\left(t, x, f_{1}(x)\right)-g\left(t, x, f_{2}(x)\right), \int_{0}^{x} g\left(t, y, f_{2}(y)\right) d y\right\rangle_{H}\right| \\
& \leq\left\|g\left(t, x, f_{1}(x)\right)\right\|_{H} \int_{0}^{\infty}\left\|g\left(t, x, f_{1}(x)\right)-g\left(t, x, f_{2}(x)\right)\right\|_{H} d x \\
& \quad+\left\|g\left(t, x, f_{1}(x)\right)-g\left(t, x, f_{2}(x)\right)\right\|_{H} \int_{0}^{\infty}\left\|g\left(t, x, f_{2}(x)\right)\right\|_{H} d x, \quad x \in[0, \infty) .
\end{aligned}
$$

It follows from Lemma 4.1 and (4.4) and (4.5) that for $t \geq 0, x \in[0, \infty)$,

$$
\begin{aligned}
\left|F\left(t, f_{1}\right)(x)-F\left(t, f_{2}\right)(x)\right| & \leq\left(\frac{p}{v q}\right)^{\frac{1}{q}}\|\hat{g}\|_{\infty}\left\|f_{1}-f_{2}\right\|_{\mathcal{v}, p}|\bar{g}(x)| \\
& +\left(\frac{p}{v q}\right)^{\frac{1}{q}}\|\bar{g}\|_{\nu, p}|\hat{g}(x)|\left|f_{1}(x)-f_{2}(x)\right| .
\end{aligned}
$$

Taking into account the last inequality, we infer that

$$
\left\|F\left(t, f_{1}\right)-F\left(t, f_{2}\right)\right\|_{\nu, p} \leq 2\left(\frac{p}{v q}\right)^{\frac{1}{q}}\|\hat{g}\|_{\infty}\|\bar{g}\|_{\nu, p}\left\|f_{1}-f_{2}\right\|_{\nu, p},
$$

concluding that $F$ is globally Lipschitz on $L_{v}^{p}$ with a Lipschitz constant independent of time $t$.

Lemma 4.7 Let $v>0$ and $p \geq 2$. Assume $g:[0, \infty) \times[0, \infty) \times \mathbb{R} \rightarrow H$ is a function satisfying the assumptions of Theorem 4.2. Then $G:[0, \infty) \times L_{v}^{p} \rightarrow \gamma\left(H, L_{v}^{p}\right)$ defined by

$$
G(t, f)[h](x)=\langle g(t, x, f(x)), h\rangle_{H}, \quad f \in L_{v}^{p}, h \in H, x, t \in[0, \infty),
$$

is well defined. Moreover, we have: 
(i) For all $t \in[0, \infty)$ and $f \in L_{\nu}^{p}$, there exists a constant $N>0$ such that

$$
\|G(t, f)\|_{\gamma\left(H, L_{v}^{p}\right)} \leq N\|\bar{g}\|_{\nu, p} .
$$

(ii) $G$ is globally Lipschitz on $L_{v}^{p}$ with a Lipschitz constant independent of time $t$.

Proof Fix $t \geq 0$ and $f \in L_{v}^{p}$. Define the function $\kappa:[0, \infty) \rightarrow H$ by

$$
\kappa(x)=g(t, x, f(x)), \quad x \in[0, \infty) .
$$

Then $G(t, f)$ can be written as

$$
G(t, f)[h](x)=\langle\kappa(x), h\rangle_{H}, \quad h \in H, x \in[0, \infty) .
$$

It follows from (4.4) that $\kappa \in L_{v}^{p}(H)$. Therefore, by Lemma 4.5, $G(t, f)$ is a $\gamma$-radonifying operator from $H$ to $L_{v}^{p}$ and so $G$ is well defined. Moreover, again by Lemma 4.5 and (4.4), we have for a constant $N>0$ independent of $\kappa$ that

$$
\|G(t, f)\|_{\gamma\left(H, L_{v}^{p}\right)} \leq N\|\bar{g}\|_{\nu, p},
$$

which gives the desired result (4.13). Finally, using Lemma 4.5 and (4.5), we have for each $t \geq 0$ and $f_{1}, f_{2} \in L_{v}^{p}$ that

$$
\left\|G\left(t, f_{1}\right)-G\left(t, f_{2}\right)\right\|_{\gamma\left(H, L_{v}^{p}\right)} \leq N\|\hat{g}\|_{\infty}\left\|f_{1}-f_{2}\right\|_{\nu, p},
$$

which implies that $G(t, \cdot)$ is globally Lipschitz on $L_{v}^{p}$ with a Lipschitz constant independent of time $t$.

Proof of Theorem 4.2 Fix $v>0$ and $p \geq 2$. The abstract form of (4.3) in the space $L_{v}^{p}$ is

$$
d r(t)=(A r(t)+F(t, r(t))) d t+G(t, r(t)) d W(t), \quad t \geq 0,
$$

where $A$ is the infinitesimal generator of the shift semigroup on $L_{v}^{p}$ (see Lemma 4.4), and $F$ and $G$ are the functions defined in Lemmas 4.6 and 4.7, respectively. Now (4.3) is of the same form as (3.1) and it follows from the previous lemmas that all the assumptions of Theorem 3.4 hold. Therefore, in view of Theorem $3.4,(4.15)$ has a unique $L_{v}^{p}$-valued continuous mild solution with the initial value $r_{0} \in L^{2}\left(\Omega, \mathcal{F}_{0}, \mathbb{P} ; L_{v}^{p}\right)$.

Example 4.8 Let us consider the function $g$ defined by

$$
\alpha(t, x)=g(x)=\sigma_{0} e^{-\lambda x}, \quad t \geq 0, x \in[0, \infty),
$$

where $\sigma_{0}, \lambda$ are constants and $\lambda>0$. This corresponds to the volatility $\sigma$ in (4.1) having the form

$$
\sigma(t, T)=g(T-t)=\sigma_{0} e^{-\lambda(T-t)}, \quad 0 \leq t \leq T<\infty .
$$


With this function $g$, (4.3) (driven by a standard one-dimensional Wiener process $W=(W(t)), t \geq 0)$ transforms to

$$
\begin{aligned}
d r(t)(x)= & \left(\frac{\partial}{\partial x} r(t)(x)+\frac{\sigma_{0}^{2}}{\lambda}\left(e^{-\lambda x}-e^{-2 \lambda x}\right)\right) d t \\
& +\sigma_{0} e^{-\lambda x} d W(t), \quad t, x \geq 0 .
\end{aligned}
$$

As $g$ satisfies all assumptions of Theorem 4.2, we infer that (4.16) has a unique $L_{v}^{p}$-valued continuous mild solution with initial value $r_{0} \in L^{2}\left(\Omega, \mathcal{F}_{0}, \mathbb{P} ; L_{v}^{p}\right)$. Moreover, by the definition of a mild solution, this unique solution is of the exact form

$$
\begin{aligned}
r(t)(x)-r_{0}(x+t)= & \frac{\sigma_{0}^{2}}{\lambda} \int_{0}^{t}\left(e^{-\lambda(x+s)}-e^{-2 \lambda(x+s)}\right) d s+\sigma_{0} \int_{0}^{t} e^{-\lambda(x+s)} d W(s) \\
= & \frac{\sigma_{0}^{2}}{2 \lambda^{2}}\left(2 e^{-\lambda x}\left(1-e^{-\lambda t}\right)-e^{-2 \lambda x}\left(1-e^{-2 \lambda t}\right)\right) \\
& +\sigma_{0} e^{-\lambda x} \int_{0}^{t} e^{-\lambda s} d W(s), \quad t, x \geq 0 .
\end{aligned}
$$

We can also consider the functions $g_{1}$ and/or $g_{2}$ defined by

$$
\begin{array}{ll}
g_{1}(x)=e^{-\lambda x} \cos (\beta x), & x \in[0, \infty), \\
g_{2}(x)=e^{-\lambda x} \sin (\beta x), & x \in[0, \infty),
\end{array}
$$

where $\lambda>0$ and $\beta \in \mathbb{R}$. As these functions obviously satisfy all the assumptions of Theorem 4.2, we infer that for each $r_{0} \in L^{2}\left(\Omega, \mathcal{F}_{0}, \mathbb{P} ; L_{v}^{p}\right)$, (4.3) with the function $g_{i}, i=1,2$, has a unique $L_{v}^{p}$-valued continuous mild solution with initial value $r_{0}$.

Example 4.9 Let us next consider the function $g$ defined by

$$
g(x, u)=e^{-\lambda x} \sin u, \quad x \in[0, \infty), u \in \mathbb{R},
$$

where $\lambda>0$. With this function $g$, (4.3) changes to

$$
\begin{aligned}
d r(t)(x)= & \left(\frac{\partial}{\partial x} r(t)(x)+e^{-\lambda x} \sin (r(t)(x)) \int_{0}^{x} e^{-\lambda y} \sin (r(t)(y)) d y\right) d t \\
& +e^{-\lambda x} \sin (r(t)(x)) d W(t), \quad x, t \in[0, \infty),
\end{aligned}
$$

where $(W(t))_{t \geq 0}$ is a standard one-dimensional Wiener process. Since the function sin is bounded and globally Lipschitz on $\mathbb{R}$, the function $g$ satisfies all the assumptions of Theorem 4.2. Thus, (4.9) has a unique $L_{v}^{p}$-valued continuous mild solution with the initial value $r_{0} \in L^{2}\left(\Omega, \mathcal{F}_{0}, \mathbb{P} ; L_{\nu}^{p}\right)$. 
Example 4.10 Suppose that $W(t), t \geq 0$, is a standard 1-dimensional Brownian motion and the function $\sigma$ in (4.1) is of the form

$$
\sigma(t, T)=\sigma_{0}(T-t), \quad 0 \leq t \leq T<\infty
$$

where $\sigma_{0}=g \in L^{p}(0, \infty)$. Since $\alpha(t, x)=\sigma(t, t+x)=\sigma_{0}(x)$ for $t \geq 0, x \in[0, \infty)$, we infer that for all $t \geq 0$,

$$
\|\alpha(t)\|_{\nu, p}^{p}=\int_{0}^{\infty}|\alpha(t, x)|^{p} e^{\nu x} d x=\int_{0}^{\infty}\left|\sigma_{0}(x)\right|^{p} e^{\nu x} d x<\infty .
$$

Hence, for every $t \geq 0, \alpha(t) \in L_{v}^{p}$. However, unless $\sigma_{0} \in W_{v}^{1,2}, \alpha(t)$ does not belong to the space $W_{v}^{1,2}$. In particular, one can expect that the problem (4.3) is not well posed in the space $W_{v}^{1,2}$ although it is well posed in the space $L_{v}^{p}$. It should not be too difficult to verify this claim.

This example shows that the HJMM equation, and hence also term structure equations, can be well posed on a Banach space where the point evaluation at $x=0$ is not well defined. It would be interesting to study different spaces than $L_{v}^{p}$, for instance, spaces with the norm defined by

$$
\|r\|:=\sup _{x \geq 0} \int_{0}^{x}|r(y)| d y .
$$

\subsection{Existence and uniqueness of solutions to the HJMM equations in weighted Sobolev spaces}

For each $v \in \mathbb{R}$ and $p \geq 1$, define $W_{v}^{1, p}$ to be the space of all functions $f \in L_{v}^{p}$ such that the weak derivative $D f$ belongs to $L_{v}^{p}$, i.e.,

$$
W_{v}^{1, p}=\left\{f \in L_{v}^{p}: D f \in L_{v}^{p}\right\}
$$

For each $v \in \mathbb{R}$ and $p \geq 1$, the space $W_{v}^{1, p}$ is a separable Banach space endowed with the norm

$$
\|f\|_{W_{\nu}^{1, p}}=\|f\|_{\nu, p}+\|D f\|_{\nu, p}, \quad f \in W_{\nu}^{1, p} .
$$

Proposition 4.11 For each $v \geq 0$ and $p \geq 1$, the space $W_{v}^{1, p}$ is continuously embedded into the space $L^{\infty}$. In particular, there exists a constant $C(\nu, p)>0$ depending on $v$ and $p$ such that

$$
\sup _{x \in[0, \infty)} e^{v x}|f(x)|^{p} \leq C(\nu, p)\|f\|_{W_{v}^{1, p}}^{p}, \quad f \in W_{v}^{1, p} .
$$

Proof Fix $v \geq 0, p \geq 1$ and $f \in W_{v}^{1, p}$. Let $\varepsilon>0$. Since

$$
\int_{0}^{\infty}|f(x)|^{p} e^{v x} d x<\infty,
$$


there exists $x_{0} \in[0, \infty)$ such that $e^{v x_{0}}\left|f\left(x_{0}\right)\right|^{p}<\varepsilon$. Consider $x \in\left[x_{0}, \infty\right)$. Then

$$
\begin{aligned}
e^{v x}|f(x)|^{p}= & e^{v x_{0}}\left|f\left(x_{0}\right)\right|^{p}+p \int_{x_{0}}^{x}|f(y)|^{p-1} D f(y) e^{v y} d y \\
& +v \int_{x_{0}}^{x}|f(y)|^{p} e^{v y} d y, \quad x \in\left[x_{0}, \infty\right) .
\end{aligned}
$$

Therefore, we have

$$
\sup _{x \in\left[x_{0}, \infty\right)} e^{\nu x}|f(x)|^{p} \leq \varepsilon+p \int_{x_{0}}^{\infty}|f(x)|^{p-1} D f(x) e^{v x} d x+v\|f\|_{\nu, p}^{p} .
$$

Using the Hölder and Young inequalities, we get

$$
\begin{aligned}
\int_{x_{0}}^{\infty}|f(x)|^{p-1} D f(x) e^{\nu x} d x & \leq\left(\int_{x_{0}}^{\infty}|f(x)|^{p} e^{\nu x} d x\right)^{\frac{p-1}{p}}\left(\int_{x_{0}}^{\infty}|D f(x)|^{p} e^{\nu x} d x\right)^{\frac{1}{p}} \\
& \leq \frac{p-1}{p}\|f\|_{\nu, p}^{p}+\frac{1}{p}\|D f\|_{\nu, p}^{p} .
\end{aligned}
$$

Taking into account the last inequality, we obtain

$$
\sup _{x \in\left[x_{0}, \infty\right)} e^{\nu x}|f(x)|^{p} \leq \varepsilon+(p-1)\|f\|_{\nu, p}^{p}+\|D f\|_{\nu, p}^{p}+v\|f\|_{\nu, p}^{p} .
$$

Similarly, we can prove the above inequality for $x \in\left[0, x_{0}\right)$. Since $\varepsilon>0$ is arbitrary, we infer that

$$
\sup _{x \in[0, \infty)} e^{v x}|f(x)|^{p} \leq(p-1)\|f\|_{\nu, p}^{p}+\|D f\|_{\nu, p}^{p}+v\|f\|_{\nu, p}^{p}
$$

which gives the desired result.

Theorem 4.12 Let $v>0$ and $p \geq 2$. Assume that $g:[0, \infty) \times[0, \infty) \times \mathbb{R} \rightarrow H$ is a continuously weakly differentiable function with respect to the second and third variables such that there exist functions $\bar{g}, \hat{g} \in W_{v}^{1, p}$ with the following properties:

(i) For all $t \in[0, \infty)$,

$$
\|g(t, x, u)\|_{H} \leq|\bar{g}(x)|, \quad u \in \mathbb{R}, x \in[0, \infty) .
$$

(ii) For all $t \in[0, \infty)$,

$$
\|g(t, x, u)-g(t, x, v)\|_{H} \leq|\hat{g}(x)||u-v|, \quad u, v \in \mathbb{R}, x \in[0, \infty) .
$$

(iii) For all $t \in[0, \infty)$,

$$
\left\|D_{x} g(t, x, u)\right\|_{H} \leq|D \bar{g}(x)|, \quad u \in \mathbb{R}, x \in[0, \infty) .
$$


(iv) For all $t \in[0, \infty)$,

$$
\left\|D_{x} g(t, x, u)-D_{x} g(t, x, v)\right\|_{H} \leq|D \hat{g}(x)||u-v|, \quad u, v \in \mathbb{R}, x \in[0, \infty) \text {. }
$$

(v) There exists a constant $K_{1}>0$ such that for all $t \in[0, \infty)$,

$$
\left\|D_{u} g(t, x, u)\right\|_{H} \leq K_{1}, \quad u \in \mathbb{R}, x \in[0, \infty)
$$

(vi) There exists a constant $K_{2}>0$ such that for all $t \in[0, \infty)$,

$$
\left\|D_{u} g(t, x, u)-D_{v} g(t, x, v)\right\|_{H} \leq K_{2}|u-v|, \quad u, v \in \mathbb{R}, x \in[0, \infty) .
$$

Above, $D_{x} g(t, x, u)$ is the first weak derivative of the function $[0, \infty) \ni x \mapsto g(t, x, u)$ when $t$ and $u$ are fixed. Similarly, $D_{u} g(t, x, u)$ is the first weak derivative of the function $\mathbb{R} \ni u \mapsto g(t, x, u)$ when $t$ and $x$ are fixed.

Then for each $r_{0} \in L^{2}\left(\Omega, \mathcal{F}_{0}, \mathbb{P} ; W_{v}^{1, p}\right)$, (4.3) with the function $g$ has a unique $W_{v}^{1, p}$-valued continuous mild solution with the initial value $r(0)=r_{0}$.

In view of Theorem 3.11, the proof follows from the following lemmas.

Lemma 4.13 For each $v \in \mathbb{R}$ and $p \geq 2$, the space $W_{v}^{1, p}$ satisfies the $H$-condition.

Similarly to the argument for Lemma 4.3, the proof follows from the fact that for each $p \geq 2$, the space $W^{1, p}$ satisfies the $H$-condition; see [13].

Lemma 4.14 For each $v \in \mathbb{R}$ and $p \geq 1$, the shift semigroup on $W_{v}^{1, p}$ is a contraction-type $C_{0}$-semigroup. Moreover, its infinitesimal generator is characterized by

$$
\mathcal{D}(A)=\left\{f \in W_{v}^{1, p}: D f \in W_{v}^{1, p}\right\}
$$

and

$$
A f=D f, \quad f \in \mathcal{D}(A) .
$$

The proof of the first part of Lemma 4.14 can be found in [37, Lemma 5.13] and the proof of the second part in [37, Lemma 5.16].

For each $v \in \mathbb{R}$ and $p \geq 1$, define $W_{v}^{1, p}(H)$ to be the space of all functions $f \in L^{p}(H)$ such that $D f \in L^{p}(H)$. For each $v \in \mathbb{R}$ and $p \geq 1, W_{v}^{1, p}(H)$ is a separable Banach space with respect to the norm

$$
\|f\|_{W_{v}^{1, p}(H)}=\|f\|_{L_{v}^{p}(H)}+\|D f\|_{L_{v}^{p}(H)} \quad f \in W_{v}^{1, p}(H) .
$$

The following proposition gives a sufficient condition under which a $W_{v}^{1, p}$-valued operator defined on $H$ is $\gamma$-radonifying. 
Lemma 4.15 For each $v>0$ and $p \geq 2$, the bounded linear operator $K: H \rightarrow W_{v}^{1, p}$ defined by

$$
K[h](x)=\langle\kappa(x), h\rangle_{H}, \quad h \in H, x \in[0, \infty),
$$

where $\kappa \in W_{v}^{1, p}(H)$, is a $\gamma$-radonifying operator. Moreover, there exists a constant $N>0$ independent of $\kappa$ such that

$$
\|K\|_{\gamma\left(H, W_{v}^{1, p}\right)} \leq N\|\kappa\|_{W_{v}^{1, p}(H)} .
$$

Similarly to the proof of Lemma 4.5, the proof follows from [13, Theorem 4.1].

Lemma 4.16 Let $v>0$ and $p \geq 1$. Assume $g:[0, \infty) \times[0, \infty) \times \mathbb{R} \rightarrow H$ is a function satisfying all the assumptions of Theorem 4.12. Then $F:[0, \infty) \times W_{v}^{1, p} \rightarrow W_{v}^{1, p}$ defined by

$$
F(t, f)(x)=\left\langle g(t, x, f(x)), \int_{0}^{x} g(t, y, f(y)) d y\right\rangle_{H}, \quad f \in W_{\nu}^{1, p}, x, t \in[0, \infty),
$$

is well defined. Moreover, we have:

(i) For every $t \in[0, \infty)$,

$$
\begin{aligned}
\|F(t, f)\|_{W_{v}^{1, p}} \leq & \|\bar{g}\|_{1}\|\bar{g}\|_{\nu, p}+3\|\bar{g}\|_{1}\|D \bar{g}\|_{\nu, p} \\
& +3 K_{1}\|\bar{g}\|_{1}\|D f\|_{\nu, p}+3\|\bar{g}\|_{\infty}\|\bar{g}\|_{\nu, p} .
\end{aligned}
$$

(ii) $F$ is Lipschitz on balls with Lipschitz constant independent of time $t$.

Proof Fix $t \geq 0$ and $f \in W_{v}^{1, p}$. In Lemma 4.6, we have already proved that $F(t, f) \in L_{v}^{p}$ under conditions (4.17) and (4.18). Let us show that $D F(t, f) \in L_{v}^{p}$. By the chain rule, we have

$$
\begin{aligned}
D F(t, f)(x)= & \left\langle D g(t, x, f(x)), \int_{0}^{x} g(t, y, f(y)) d y\right\rangle_{H} \\
& +\langle g(t, x, f(x)), g(t, x, f(x))\rangle_{H} .
\end{aligned}
$$

Since the functions $g(t, x, f(x)), D g(t, x, f(x))$ and $h(x):=\int_{0}^{x} g(t, y, f(y))$ are measurable, $D F(t, f)$ is measurable. Moreover, by the Cauchy-Schwarz inequality, we obtain for each $x \in[0, \infty)$ that

$$
\begin{aligned}
|D F(t, f)(x)| \leq & \|D g(t, x, f(x))\|_{H} \int_{0}^{\infty}\|g(t, x, f(x))\|_{H} d x \\
& +\|g(t, x, f(x))\|_{H}\|g(t, x, f(x))\|_{H} .
\end{aligned}
$$

Using (4.17) and Lemma 4.1, we obtain

$$
|D F(t, f)(x)| \leq\|\bar{g}\|_{1}\|D g(t, x, f(x))\|_{H}+|\bar{g}(x)|^{2}, \quad x \in[0, \infty) .
$$


Note that

$$
D g(t, x, f(x))=D_{x} g(t, x, f(x))+D_{u} g(t, x, f(x)) D f(x), \quad x \in[0, \infty) .
$$

Therefore, by (4.19) and (4.21), we get for each $x \in[0, \infty)$ that

$$
\begin{aligned}
\|D g(t, x, f(x))\|_{H} & \leq\left\|D_{x} g(t, x, f(x))\right\|_{H}+\left\|D_{u} g(t, x, f(x))\right\|_{H}|D f(x)| \\
& \leq|D \bar{g}(x) t|+K_{1}|D f(x)| .
\end{aligned}
$$

Taking into account the last inequality, we obtain

$$
|D F(t, f)(x)| \leq\|\bar{g}\|_{1}|D \bar{g}(x)|+K_{1}\|\bar{g}\|_{1}|D f(x)|+|\bar{g}(x)|^{2}, \quad x \in[0, \infty) .
$$

Thus, by the last inequality and Proposition 4.11, we infer that $\int_{0}^{\infty}|D F(t, f)|^{p} e^{\nu x} d x \leq 3^{p}\|\bar{g}\|_{1}^{p}\|D \bar{g}\|_{\nu, p}^{p}+3^{p} K_{1}^{p}\|\bar{g}\|_{1}^{p}\|D f\|_{\nu, p}^{p}+3^{p}\|\bar{g}\|_{\infty}^{p}\|\bar{g}\|_{\nu, p}^{p}$.

Therefore $D F(t, f) \in L_{v}^{P}$ and hence $F$ is well defined. Moreover, it follows from (4.10) and the last inequality that

$$
\begin{aligned}
\|F(t, f)\|_{W_{v}^{1, p} \leq} \leq & \|\bar{g}\|_{1}\|\bar{g}\|_{\nu, p}+3\|\bar{g}\|_{1}\|D \bar{g}\|_{\nu, p} \\
& +3 K_{1}\|\bar{g}\|_{1}\|D f\|_{\nu, p}+3\|\bar{g}\|_{\infty}\|\bar{g}\|_{\nu, p},
\end{aligned}
$$

which gives the desired conclusion (4.23).

Let us now prove that $F$ is locally Lipschitz on balls. Fix $t \geq 0$ and $f_{1}, f_{2} \in W_{v}^{1, p}$. Note that

$$
\begin{aligned}
& F\left(t, f_{1}\right)(x)-F\left(t, f_{2}\right)(x) \\
& =\left\langle g\left(t, x, f_{1}(x)\right)-g\left(t, x, f_{2}(x)\right), \int_{0}^{x} g\left(t, y, f_{2}(y)\right) d y\right\rangle_{H} \\
& \quad+\left\langle g\left(t, x, f_{1}(x)\right), \int_{0}^{x}\left(g\left(t, y, f_{1}(y)\right)-g\left(t, y, f_{2}(y)\right)\right) d y\right\rangle_{H}, \quad x \in[0, \infty) .
\end{aligned}
$$

By the Cauchy-Schwarz inequality, we have

$$
\begin{aligned}
& \left|F\left(t, f_{1}\right)(x)-F\left(t, f_{2}\right)(x)\right| \\
& \leq\left\|g\left(t, x, f_{1}(x)\right)-g\left(t, x, f_{2}(x)\right)\right\|_{H} \int_{0}^{\infty}\left\|g\left(t, x, f_{2}(x)\right)\right\|_{H} d x \\
& \quad+\left\|g\left(t, x, f_{1}(x)\right)\right\|_{H} \int_{0}^{\infty}\left\|g\left(t, x, f_{1}(x)\right)-g\left(t, x, f_{2}(x)\right)\right\| d x, \quad x \in[0, \infty) .
\end{aligned}
$$

Using (4.17) and (4.18), we obtain for each $x \in[0, \infty)$ that

$$
\begin{aligned}
\left|F\left(t, f_{1}\right)(x)-F\left(t, f_{2}\right)(x)\right| \leq & |\hat{g}(x)|\left|f_{1}(x)-f_{2}(x)\right| \int_{0}^{\infty}|\bar{g}(x)| d x \\
& +|\bar{g}(x)| \int_{0}^{\infty}|\hat{g}(x)|\left|f_{1}(x)-f_{2}(x)\right| d x .
\end{aligned}
$$


It follows from Lemma 4.1 and Proposition 4.11 that

$$
\begin{aligned}
\left|F\left(t, f_{1}\right)(x)-F\left(t, f_{2}\right)(x)\right| \leq & \|\bar{g}\|_{1}\left|\hat{g}(x) \| f_{1}(x)-f_{2}(x)\right| \\
& +\left\|f_{1}-f_{2}\right\|_{1}\|\hat{g}\|_{\infty}|\bar{g}(x)|, \quad x \in[0, \infty) .
\end{aligned}
$$

Taking into account the last inequality and Proposition 4.11, we infer that

$$
\begin{aligned}
\left\|F\left(t, f_{1}\right)-F\left(t, f_{2}\right)\right\|_{\nu, p} \leq & 2\|\bar{g}\|_{1}\|\hat{g}\|_{\infty}\left\|f_{1}-f_{2}\right\|_{\nu, p} \\
& +2\|\hat{g}\|_{\infty}\|\bar{g}\|_{\nu, p}\left\|f_{1}-f_{2}\right\|_{1} .
\end{aligned}
$$

By the chain rule, we get for $x \in[0, \infty)$ that

$$
\begin{aligned}
D\left(F\left(t, f_{1}\right)(x)-F\left(t, f_{2}\right)(x)\right)= & \left\langle D g\left(t, x, f_{1}(x)\right), \int_{0}^{x} g\left(t, y, f_{1}(y)\right) d y\right\rangle_{H} \\
& +\left\langle g\left(t, x, f_{1}(x)\right), g\left(t, x, f_{1}(x)\right)\right\rangle_{H} \\
& -\left\langle D g\left(t, x, f_{2}(x)\right), \int_{0}^{x} g\left(t, y, f_{2}(y)\right) d y\right\rangle_{H} \\
& -\left\langle g\left(t, x, f_{2}(x)\right), g\left(t, x, f_{2}(x)\right)\right\rangle_{H} .
\end{aligned}
$$

Note that

$$
\begin{aligned}
D & \left(F\left(t, f_{1}\right)(x)-F\left(t, f_{2}\right)(x)\right) \\
= & \left\langle D g\left(t, x, f_{1}(x)\right), \int_{0}^{x}\left(g\left(t, y, f_{1}(y)\right)-g\left(t, y, f_{2}(y)\right)\right) d y\right\rangle_{H} \\
& +\left\langle D g\left(t, x, f_{1}(x)\right)-D g\left(t, x, f_{2}(x)\right), \int_{0}^{x} g\left(t, y, f_{2}(y)\right) d x\right\rangle_{H} \\
& +\left\langle g\left(t, x, f_{1}(x)\right), g\left(t, x, f_{1}(x)\right)-g\left(t, x, f_{2}(x)\right)\right\rangle_{H} \\
& +\left\langle g\left(t, x, f_{1}(x)\right)-g\left(t, x, f_{2}(x)\right), g\left(t, x, f_{2}(x)\right)\right\rangle_{H}, \quad x \in[0, \infty) .
\end{aligned}
$$

Using the Cauchy-Schwarz inequality and (4.17) and (4.18), we obtain

$$
\begin{aligned}
& \left|D\left(F\left(t, f_{1}\right)(x)-F\left(t, f_{2}\right)(x)\right)\right| \\
& \leq\left\|D g\left(t, x, f_{1}(x)\right)\right\|_{H} \int_{0}^{\infty}|\hat{g}(x)|\left|f_{1}(x)-f_{2}(x)\right| d x \\
& \quad+\left\|D g\left(t, x, f_{1}(x)\right)-D g\left(t, x, f_{2}(x)\right)\right\|_{H} \int_{0}^{\infty}|\bar{g}(x)| d x \\
& \quad+2|\bar{g}(x)||\hat{g}(x)|\left|f_{1}(x)-f_{2}(x)\right|, \quad x \in[0, \infty) .
\end{aligned}
$$


By Lemma 4.1 and Proposition 4.11, we get for $x \in[0, \infty)$ that

$$
\begin{aligned}
\left|D\left(F\left(t, f_{1}\right)(x)-F\left(t, f_{2}\right)(x)\right)\right| \leq & \|\hat{g}\|_{\infty}\left\|f_{1}-f_{2}\right\|_{1}\left\|D g\left(t, x, f_{1}(x)\right)\right\|_{H} \\
& +\|\bar{g}\|_{1}\left\|D g\left(t, x, f_{1}(x)\right)-D g\left(t, x, f_{2}(x)\right)\right\|_{H} \\
& +2 \mid \bar{g}(x)\|\hat{g}(x)\| f_{1}(x)-f_{2}(x) .
\end{aligned}
$$

Note that

$$
\begin{array}{ll}
D g\left(t, x, f_{1}(x)\right)=D_{x} g\left(t, x, f_{1}(x)\right)+D_{u} g\left(t, x, f_{1}(x)\right) D f_{1}(x), & x \in[0, \infty), \\
D g\left(t, x, f_{2}(x)\right)=D_{x} g\left(t, x, f_{2}(x)\right)+D_{v} g\left(t, x, f_{2}(x)\right) D f_{2}(x), & x \in[0, \infty) .
\end{array}
$$

Thus

$$
\begin{aligned}
& D g\left(t, x, f_{1}(x)\right)-D g\left(t, x, f_{2}(x)\right) \\
& =D_{x} g\left(t, x, f_{1}(x)\right)-D_{x} g\left(t, x, f_{2}(x)\right)+D_{u} g\left(t, x, f_{1}(x)\right)\left(D f_{1}(x)-D f_{2}(x)\right) \\
& \quad+\left(D_{u} g\left(t, x, f_{1}(x)\right)-D_{v} g\left(t, x, f_{2}(x)\right)\right) D f_{2}(x), \quad x \in[0, \infty) .
\end{aligned}
$$

Using the Cauchy-Schwarz inequality, we obtain

$$
\begin{aligned}
& \left\|D g\left(t, x, f_{1}(x)\right)-D g\left(t, x, f_{2}(x)\right)\right\|_{H} \\
& \leq\left\|D_{x} g\left(t, x, f_{1}(x)\right)-D_{x} g\left(t, x, f_{2}(x)\right)\right\|_{H} \\
& \quad+\left\|D_{u} g\left(t, x, f_{1}(x)\right)\right\|_{H}\left|D f_{1}(x)-D f_{2}(x)\right| \\
& \quad+\left\|D_{u} g\left(t, x, f_{1}(x)\right)-D_{v} g\left(t, x, f_{2}(x)\right)\right\|_{H}\left|D f_{2}(x)\right| .
\end{aligned}
$$

It follows from (4.20)-(4.22) that for $x \in[0, \infty)$,

$$
\begin{aligned}
& \left\|D g\left(t, x, f_{1}(x)\right)-D g\left(t, x, f_{2}(x)\right)\right\|_{H} \\
& \leq|D \hat{g}(x)|\left|f_{1}(x)-f_{2}(x)\right|+K_{1}\left|D f_{1}(x)-D f_{2}(x)\right| \\
& \quad+K_{2}\left|f_{1}(x)-f_{2}(x)\right|\left|D f_{2}(x)\right| .
\end{aligned}
$$

Taking into account (4.24) and the last inequality, we obtain for each $x \in[0, \infty)$ that

$$
\begin{aligned}
& \left|D\left(F\left(t, f_{1}\right)(x)-F\left(t, f_{2}\right)(x)\right)\right| \\
& \leq\|\hat{g}\|_{\infty}\left\|f_{1}-f_{2}\right\|_{1}|D \bar{g}(x)|+K_{1}\|\hat{g}\|_{\infty}\left\|f_{1}-f_{2}\right\|_{1}\left|D f_{1}(x)\right| \\
& \quad+\|\bar{g}\|_{1}\left|D \hat { g } ( x ) \left\|f_{1}(x)-f_{2}(x)\left|+K_{1}\|\bar{g}\|_{1}\right| D f_{1}(x)-D f_{2}(x) \mid\right.\right. \\
& \quad+K_{2}\|\bar{g}\|_{1}\left|f_{1}(x)-f_{2}(x)\right|\left|D f_{2}(x)\right|+2|\bar{g}(x)||\hat{g}(x)|\left|f_{1}(x)-f_{2}(x)\right| .
\end{aligned}
$$


Using the last inequality and Proposition 4.11, we infer that

$$
\begin{aligned}
\| D & \left(F\left(t, f_{1}\right)-F\left(t, f_{2}\right)\right) \|_{\nu, p} \\
\leq & 6\|\hat{g}\|_{\infty}\left\|f_{1}-f_{2}\right\|_{1}\|D \bar{g}\|_{\nu, p}+6 K_{1}\|\hat{g}\|_{\infty}\left\|f_{1}-f_{2}\right\|_{1}\left\|D f_{1}\right\|_{\nu, p} \\
& +6\|\bar{g}\|_{1}\|D \hat{g}\|_{\nu, p}\left\|f_{1}-f_{2}\right\|_{\infty}+6 K_{1}\|\bar{g}\|_{1}\left\|D f_{1}-D f_{2}\right\|_{\nu, p} \\
& +6 K_{2}\|\bar{g}\|_{1}\left\|f_{1}-f_{2}\right\|_{\infty}\left\|D f_{2}\right\|_{\nu, p}+6\|\hat{g}\|_{\infty}\|\bar{g}\|_{\infty}\left\|f_{1}-f_{2}\right\|_{\nu, p}^{p} .
\end{aligned}
$$

It follows from (4.25) and (4.27) that $F$ is Lipschitz on balls.

Lemma 4.17 Let $v>0$ and $p \geq 1$. Suppose $g:[0, \infty) \times[0, \infty) \times \mathbb{R} \rightarrow H$ satisfies all the assumptions of Theorem 4.12. Then $G:[0, \infty) \times W_{v}^{1, p} \rightarrow \gamma\left(H, W_{v}^{1, p}\right)$ defined by

$$
G(t, f)[h](x)=\langle g(t, x, f(x)), h\rangle_{H}, \quad f \in W_{v}^{1, p}, h \in H, x, t \in[0, \infty),
$$

is well defined. Moreover, we have:

(i) For every $t \in[0, \infty)$,

$$
\|G(t, f)\|_{\gamma\left(H, W_{v}^{1, p}\right)} \leq N\left(\|\bar{g}\|_{\mathcal{v}, p}+2\|D \bar{g}\|_{\nu, p}+2 K_{1}\|D f\|_{\nu, p}\right), \quad f \in W_{v}^{1, p} .
$$

(ii) $G$ is Lipschitz on balls with Lipschitz constant independent of time $t$.

Proof Fix $t \geq 0$ and $f \in W_{v}^{1, p}$. Define the function $\kappa:[0, \infty) \rightarrow H$ by

$$
\kappa(x)=g(t, x, f(x)), \quad x \in[0, \infty) .
$$

Then we can write $G(t, f)$ as

$$
G(t, f)[h](x)=\langle\kappa(x), h\rangle_{H}, \quad h \in H, x \in[0, \infty) .
$$

It follows from (4.17) that $\kappa \in L_{v}^{p}(H)$. Moreover, (4.24) implies that $D \kappa \in L_{v}^{p}(H)$. Therefore, by Lemma $4.15, G(t, f)$ is a $\gamma$-radonifying operator from $H$ into $W_{v}^{1, p}$, and thus $G$ is well defined. Furthermore, again by Lemma 4.15 and (4.17) and (4.24), we obtain

$$
\|G(t, f)\|_{\gamma\left(H, W_{v}^{1, p}\right)} \leq N\left(\|\bar{g}\|_{v, p}+2\|D \bar{g}\|_{\nu, p}+2 K_{1}\|D f\|_{\nu, p}\right)
$$

which gives the desired result (4.28). Finally, we prove that $G$ is Lipschitz on balls. Fix $t \geq 0$ and $f_{1}, f_{2} \in W_{v}^{1, p}$. Define the function $\lambda:[0, \infty) \rightarrow H$ by

$$
\lambda(x)=g\left(t, x, f_{1}(x)\right)-g\left(t, x, f_{2}(x)\right), \quad x \in[0, \infty) .
$$

Then

$$
\left(G\left(t, f_{1}\right)-G\left(t, f_{2}\right)\right)[h](x)=\langle\lambda(x), h\rangle_{H}, \quad x \in[0, \infty), h \in H .
$$


By (4.18) and Proposition 4.11, we get

$$
\begin{aligned}
\int_{0}^{\infty}\|\lambda(x)\|_{H}^{p} e^{v x} d x & \leq \int_{0}^{\infty}|\hat{g}(x)|^{p}\left|f_{1}(x)-f_{2}(x)\right|^{p} e^{\nu x} d x \\
& \leq\|\hat{g}\|_{\infty}^{p}\left\|f_{1}-f_{2}\right\|_{v, p}^{p} .
\end{aligned}
$$

Moreover, by (4.26) and Proposition 4.11, we obtain

$$
\begin{aligned}
\int_{0}^{\infty}\|D \lambda(x)\|_{H}^{p} e^{v x} d x \leq & 3^{p}\left\|f_{1}-f_{2}\right\|_{\infty}^{p}\|D \hat{g}\|_{\nu, p}^{p}+3^{p} K_{1}^{p}\left\|D f_{1}-D f_{2}\right\|_{\nu, p}^{p} \\
& +3^{p} K_{2}^{p}\left\|f_{1}-f_{2}\right\|_{\infty}^{p}\left\|D f_{2}\right\|_{\nu, p}^{p} .
\end{aligned}
$$

It follows from Lemma 4.15 and (4.29) and (4.30) that $G$ is Lipschitz on balls.

Remark 4.18 One can easily check that the functions in Examples 4.8 and 4.9 satisfy all the assumptions of Theorem 4.12. Therefore, the HJMM equation with one of these functions has a unique $W_{v}^{1, p}$-valued continuous mild solution.

Remark 4.19 The elements of $W_{v}^{1, p}$ are $\alpha$-Hölder-continuous functions for $\alpha<1-\frac{1}{p}$, and hence for each $p \geq 2$, the solution to the HJMM equation in the space $W_{v}^{1, p}$ is more regular than the solution in the space $W_{v}^{1,2}$. On the other hand, in the spaces $C^{\alpha}$ of $\alpha$-Hölder-continuous functions, one cannot define an Itô integral, and hence these spaces are not suitable for our purposes.

\subsection{Existence and uniqueness of invariant measures for the HJMM equations in weighted Lebesgue spaces}

In this section, we prove the existence of a unique invariant measure for the HJMM equation (with time-independent coefficients) in the spaces $L_{\nu}^{p}, p \geq 2$. Let us start by presenting the following natural conclusion of Theorem 4.2.

Corollary 4.20 Let $v>0$ and $p \geq 2$. Assume that for a time-independent function $g:[0, \infty) \times \mathbb{R} \rightarrow H$, all the assumptions of Theorem 4.2 are satisfied. Then for each $r_{0} \in L^{2}\left(\Omega, \mathcal{F}_{0}, \mathbb{P} ; L_{v}^{p}\right)$, the HJMM equation with the function $g$ has a unique $L_{v}^{p}$-valued continuous mild solution $r$ with the initial value $r(0)=r_{0}$. Moreover, the solution is a Markov process.

Theorem 4.21 Assume that all the assumptions of Theorem 4.2 are satisfied for a time-independent function $g:[0, \infty) \times \mathbb{R} \rightarrow H$. If $v>0$ and $p \geq 2$ are such that

$$
2\left(\frac{p}{v q}\right)^{\frac{1}{q}}\|\hat{g}\|_{\infty}\|\bar{g}\|_{\nu, p}+(p-1) N^{2}\|\hat{g}\|_{\infty}^{2}<\frac{v}{2},
$$

where $\frac{1}{p}+\frac{1}{q}=1$, then the HJMM equation with the function $g$ has a unique invariant probability measure in the space $L_{v}^{p}$. 
Proof Assume that $F: L_{v}^{p} \rightarrow L_{v}^{p}$ and $G: L_{v}^{p} \rightarrow \gamma\left(H, L_{v}^{p}\right)$ are defined by

$$
F(f)(x)=\left\langle g(x, f(x)), \int_{0}^{x} g(y, f(y)) d y\right\rangle_{H}, \quad f \in L_{v}^{p}, x \in[0, \infty),
$$

and

$$
G(f)[h](x)=\langle g(x, f(x)), h\rangle_{H}, \quad f \in L_{v}^{p}, x \in[0, \infty), h \in H .
$$

Then the abstract form of the HJMM equation (with the function $g$ ) in the space $L_{v}^{p}$ is

$$
d r(t)=(A r(t)+F(r(t))) d t+G(r(t)) d W(t), \quad t \geq 0
$$

where $A$ is the infinitesimal generator of the shift semigroup on $L_{v}^{p}$. By Theorem 3.17, it is sufficient to show that there exist constants $\omega>0$ and $n_{0} \in \mathbb{N}$ such that for all $n \geq n_{0}$ and $f_{1}, f_{2} \in L_{\nu}^{p}$,

$$
\begin{aligned}
& {\left[A_{n}\left(f_{1}-f_{2}\right)+F\left(f_{1}\right)-F\left(f_{2}\right), f_{1}-f_{2}\right]_{v, p}+(p-1)\left\|G\left(f_{1}\right)-G\left(f_{2}\right)\right\|_{\gamma\left(H, L_{v}^{p}\right)}^{2}} \\
& \leq-\omega\left\|f_{1}-f_{2}\right\|_{v, p}^{2},
\end{aligned}
$$

where $[\cdot, \cdot]_{v, p}$ is the semi-inner product on $L_{v}^{p}$ given by (see [10])

$$
[f, g]_{v, p}=\|g\|_{\nu, p}^{2-p} \int_{0}^{\infty} f(x) g(x)|g(x)|^{p-2} e^{v x} d x, \quad f, g \in L_{v}^{p},
$$

and $A_{n}$ is the Yosida approximation of $A$. We prove (4.32) in the following few steps.

Step 1. Fix $f_{1}, f_{2} \in L_{v}^{p}$. Then by (4.12), we have

$$
\begin{aligned}
\left|F\left(f_{1}\right)(x)-F\left(f_{2}\right)(x)\right| \leq & \left(\frac{p}{v q}\right)^{\frac{1}{q}}\|\hat{g}\|_{\infty}\left\|f_{1}-f_{2}\right\|_{\nu, p}|\bar{g}(x)| \\
& +\left(\frac{p}{v q}\right)^{\frac{1}{q}}\|\bar{g}\|_{\nu, p}|\hat{g}(x)|\left|f_{1}(x)-f_{2}(x)\right|, \quad x \in[0, \infty) .
\end{aligned}
$$

Taking into account the last inequality, we obtain

$$
\begin{aligned}
& {\left[F\left(f_{1}\right)-F\left(f_{2}\right), f_{1}-f_{2}\right]_{v, p}} \\
& \leq\left\|f_{1}-f_{2}\right\|_{v, p}^{2-p} \int_{0}^{\infty}\left|F\left(f_{1}\right)(x)-F\left(f_{2}\right)(x)\right|\left|f_{1}(x)-f_{2}(x)\right|^{p-1} e^{v x} d x \\
& \leq\left(\frac{p}{v q}\right)^{\frac{1}{q}}\|\hat{g}\|_{\infty}\left\|f_{1}-f_{2}\right\|_{\nu, p}^{3-p} \int_{0}^{\infty}|\bar{g}(x)|\left|f_{1}(x)-f_{2}(x)\right|^{p-1} e^{v x} d x \\
& \quad+\left(\frac{p}{v q}\right)^{\frac{1}{q}}\|\bar{g}\|_{v, p}\left\|f_{1}-f_{2}\right\|_{v, p}^{2-p}\|\hat{g}\|_{\infty}\left\|f_{1}-f_{2}\right\|_{\nu, p}^{p} .
\end{aligned}
$$


Using the Hölder inequality, we get

$$
\int_{0}^{\infty}|\bar{g}(x)|\left|f_{1}(x)-f_{2}(x)\right|^{p-1} e^{\nu x} d x \leq\|\bar{g}\|_{\nu, p}\left\|f_{1}-f_{2}\right\|_{\nu, p}^{p-1} .
$$

By the last inequality, we deduce that

$$
\left[F\left(f_{1}\right)-F\left(f_{2}\right), f_{1}-f_{2}\right]_{\nu, p} \leq 2\left(\frac{p}{v q}\right)^{\frac{1}{q}}\|\hat{g}\|_{\infty}\|\bar{g}\|_{\nu, p}\left\|f_{1}-f_{2}\right\|_{\nu, p}^{2} .
$$

Step 2. For each $t \geq 0$, define the operator $P(t): L_{v}^{p} \rightarrow L_{v}^{p}$ by

$$
P(t) f=e^{\frac{v t}{p}} S(t) f, \quad f \in L_{v}^{p},
$$

where $\{S(t)\}_{t \geq 0}$ is the shift semigroup on $L_{v}^{p}$. It is obvious that the family $(P(t))_{t \geq 0}$ of these operators is a contraction $C_{0}$-semigroup on $L_{v}^{p}$ and its infinitesimal generator is

$$
B=\frac{\nu I}{p}+A
$$

By [49, Theorem 4.3 (b)], $B$ is dissipative and so for all $f \in \mathcal{D}(B)$ and $f^{*} \in F(f)$, we have $\left\langle B f, f^{*}\right\rangle \leq 0$, where

$$
F(f)=\left\{f^{*} \in\left(L_{v}^{p}\right)^{*}:\left\langle f, f^{*}\right\rangle=\|f\|^{2}=\left\|f^{*}\right\|^{2}\right\},
$$

where $\left(L_{v}^{p}\right)^{*}$ is the dual space of $L_{v}^{p}$ and by [10], $\left(L_{v}^{p}\right)^{*}=L_{v}^{q}$. By the definition of the semi-inner product, see Definition 3.18, we have

$$
[f, g]_{v, p}=\left\langle f, g^{*}\right\rangle, \quad f \in L_{v}^{p}, g^{*} \in\left(L_{v}^{p}\right)^{*},
$$

and $\left\langle g, g^{*}\right\rangle=\|g\|^{2}$, i.e., $g^{*} \in\left(L_{\nu}^{p}\right)^{*}$. Therefore, $\left\langle B f, f^{*}\right\rangle=[B f, f]_{v, p}$ and so we get $[B f, f]_{v, p} \leq 0$. Note that

$$
A_{n}=n A(n I-A)^{-1}=n\left(B-\frac{v}{p} I\right)\left(n I+\frac{v}{p} I-B\right)^{-1} .
$$

Let $\omega_{2}:=\frac{-v}{p}$ and $k:=n-\omega_{2}$. Then we obtain

$$
A_{n}=\left(\omega^{2}+k \omega_{2}\right)(k I-B)^{-1}+\left(1+\frac{\omega_{2}}{k}\right) B_{k} .
$$

Therefore, we get

$$
\left[A_{n} f, f\right]_{\nu, p} \leq\left(\omega_{2}^{2}+k \omega_{2}\right)\left\|(k I-B)^{-1}\right\|\|f\|_{\nu, p}^{2} .
$$

By [49, Theorem 3.1], $\left\|(k I-B)^{-1}\right\| \leq \frac{1}{k}$. Hence

$$
\left[A_{n} f, f\right]_{v, p} \leq \frac{\omega_{2}^{2}+k \omega_{2}}{k}\|f\|_{v, p}^{2} .
$$


Therefore, we infer that

$$
\left[A_{n}\left(f_{1}-f_{2}\right), f_{1}-f_{2}\right]_{v, p} \leq \frac{-v n}{n p+v}\left\|f_{1}-f_{2}\right\|_{v, p}^{2}, \quad f_{1}, f_{2} \in L_{v}^{p} .
$$

Step 3. Taking into account (4.14), (4.33) and (4.34), we obtain

$$
\begin{aligned}
& {\left[A_{n}\left(f_{1}-f_{2}\right)+F\left(f_{1}\right)-F\left(f_{2}\right), f_{1}-f_{2}\right]_{\nu, p}+(p-1)\left\|G\left(f_{1}\right)-G\left(f_{2}\right)\right\|_{\gamma\left(H, L_{v}^{p}\right)}^{2}} \\
& \leq C_{n}\left\|f_{1}-f_{2}\right\|_{\nu, p}^{2},
\end{aligned}
$$

where $C_{n}=2\left(\frac{p}{v q}\right)^{\frac{1}{q}}\|\hat{g}\|_{\infty}\|\bar{g}\|_{\nu, p}+(p-1) N^{2}\|\hat{g}\|_{\infty}^{2}+\frac{-v n}{n p+v}$. Since (4.31) gives

$$
C_{n} \longrightarrow 2\left(\frac{p}{v q}\right)^{\frac{1}{q}}\|\hat{g}\|_{\infty}\|\bar{g}\|_{\nu, p}+(p-1) N^{2}\|\hat{g}\|_{\infty}^{2}-\frac{v}{p}=C<0,
$$

there exists $n_{0} \in \mathbb{N}$ such that for all $\omega \in(0,-C)$,

$$
C_{n} \leq-\omega, \quad n \geq n_{0} .
$$

Therefore, (4.32) holds for any $\omega \in(0,-C)$.

Remark 4.22 Consider the HJMM equation in Example 4.8. By Theorem 4.21, for each $v>0$ and $p \geq 2$, (4.16) has a unique invariant probability measure in the space $L_{v}^{p}$.

Remark 4.23 There are many papers on the existence of invariant measures for the HJMM equations. In addition to the already mentioned papers [53, 54], one should also list [29, 41].

Remark 4.24 It would be interesting to investigate the uniqueness of an invariant measure for the HJMM equation in the weighted Sobolev spaces. A use of Malliavin calculus could prove to be essential here. In this context, one should mention two papers. The first one by Baudoin and Teichmann [1] is about hypoellipticity for finite-dimensional realisations of the HJMM equations, whose generalisation could provide a first step in proving the uniqueness. The second one by Hairer and Mattingly [33] shows how to use the hypoellipticity for (infinite-dimensional) parabolic SPDEs in conjunction with an asymptotic strong Feller property in order to prove the uniqueness of an invariant measure.

\subsection{An extension of the HJMM model}

Klein et al. [36] extended the HJMM model by an additional constituent which is not absolutely continuous in terms of the maturity $T$. Let us fix a finite time horizon $T^{*}$ and a probability measure $\mathbb{Q}$ which is equivalent to the measure $\mathbb{P} \mid \mathcal{F}_{T^{*}}$. Assume that $W$ and $V$ are $d$ - and 1-dimensional $\mathbb{Q}$-independent $\mathbb{Q}$-Brownian motions. Consider the filtration $\left(\mathcal{F}_{t}\right)_{t \geq 0}$ defined by

$$
\mathcal{F}_{t}=\sigma(W(s): 0 \leq s \leq t, V(u): u \geq 0)
$$


which is the completion of the initial enlargement of the natural filtration of $W$ with the full path of $V$. Klein et al. [36] assume that bond prices are given by

$$
P(t, T)=\exp \left(-\int_{t}^{T} g(t, u) d V(u)-\int_{t}^{T} f(t, u) d u\right), \quad 0 \leq t \leq T \leq T^{*},
$$

where for each $T \leq T^{*}$, the processes $f(t, T)$ and $g(t, T), t \in[0, T]$, satisfy the stochastic differential equations

$$
\begin{array}{ll}
f(t, T)=f(0, T)+\int_{0}^{t} a(s, T) d s+\int_{0}^{t} b(s, T) d W(s), & 0 \leq t \leq T, \\
g(t, T)=g(0, T)+\int_{0}^{t} c(s, T) d s+\int_{0}^{t} d(s, T) d W(s), & 0 \leq t \leq T .
\end{array}
$$

Remark 4.25 If $g \equiv 0$, this model includes the classical HJMM framework [34].

Let us recall, see [36], that $\mathbb{Q}$ is an equivalent local martingale measure (ELMM) if the process $\left(\frac{P(t, T)}{P\left(t, T^{*}\right)}\right)_{0 \leq t \leq T}$ is a local martingale for all $T \in\left[0, T^{*}\right]$. Let us set

$$
\begin{aligned}
& A(t, T):=\int_{T}^{T^{*}} a(t, u) d u, \quad B(t, T):=\int_{T}^{T^{*}} b(t, u) d u, \quad t \in[0, T], \\
& C(t, T):=\int_{T}^{T^{*}} c(t, u) d V(u), \quad D(t, T):=\int_{T}^{T^{*}} d(t, u) d V(u), \quad t \in[0, T] .
\end{aligned}
$$

Proposition 4.26 [36] Denote by $\Phi$ the optional sigma-algebra on $\Omega \times \mathbb{R}_{+}$. Assume that $a, b, c$ and $d$ are $\Phi \otimes \mathcal{B}\left(\mathbb{R}_{+}\right)$-measurable. Moreover, assume that ${ }^{1}$

$$
\begin{aligned}
& \int_{0}^{T^{*}} \int_{0}^{T^{*}} 1_{\{s \leq t\}}(|a(s, t)|+c(s, t) \mid) d s d t<\infty, \\
& \sup _{0 \leq s \leq t \leq T^{*}}(|b(s, t)|+|d(s, t)|)<\infty .
\end{aligned}
$$

Then $\mathbb{Q}$ is an ELMM if and only if for all $T \leq T^{*}$,

$$
A(t, T)+C(t, T)+\frac{1}{2}\left(\|B(t, T)\|^{2}+\|D(t, T)\|^{2}\right)=0 \quad d \mathbb{Q} \otimes d t \text {-a.s. }
$$

Example 4.27 Let us suppose that the process $g$ is of the special form

$$
g(t, u)=\int_{0}^{t} c(s, u) d s+W(t), \quad 0 \leq t \leq u \leq T^{*} .
$$

\footnotetext{
${ }^{1}$ As compared to [36], we added the indicator function $1_{\{s \leq t\}}$ in the first condition below. We also believe that the second condition should be replaced by $\sup _{0 \leq t \leq T \leq T^{*}} \int_{0}^{t}\left(|b(s, t)|^{2}+|d(s, t)|^{2}\right) d s<\infty$. This should be investigated further.
} 
Assume that $c(t, u)$ and $f(t, u)$ are deterministic functions which are bounded and continuous. Moreover, $f$ is differentiable with respect to the first variable. Assume that $c(0, T)=f(0, T)=0$ for all $T \geq 0$. Therefore, we have $d(t, T)=1$, $a(t, T)=f^{\prime}(t, T)$ and $b(t, T)=0$. Thus, by the drift condition (4.37), we have

$$
\int_{T}^{T^{*}} c(t, u) d V(u)+\int_{T}^{T^{*}} f^{\prime}(t, u) d u=\frac{1}{2}\left(V\left(T^{*}\right)-V(T)\right)^{2} .
$$

By the Itô formula, we obtain

$$
\left(V\left(T^{*}\right)-V(T)\right)^{2}=\int_{T^{*}}^{T} 2\left(V(u)-V\left(T^{*}\right)\right) d V(u)+\int_{T^{*}}^{T} d u .
$$

Therefore, (4.37) holds if and only if

$$
c(t, u)=V(u)-V\left(T^{*}\right) \quad \text { and } \quad f^{\prime}(t, u)=\frac{1}{2}, \quad \text { for all } 0 \leq t \leq u \leq T^{*} .
$$

If the processes $f$ and $g$ satisfy the term structure equations (4.35) and (4.36), then the corresponding Musiela parametrization processes $r_{1}$ and $r_{2}$ defined by

$$
r_{1}(t, x)=f(t, t+x), \quad r_{2}(t, x)=g(t, t+x), \quad x \geq 0,
$$

should solve the HJMM equations

$$
\left\{\begin{array}{l}
d r_{1}(t)=\left(\frac{d}{d x} r_{1}(t)+\alpha_{1}(r(t))\right) d t+\sigma_{1}(r(t)) d W(t), \\
r(0)=r_{0}
\end{array}\right.
$$

and

$$
\left\{\begin{array}{l}
d r_{2}(t)=\left(\frac{d}{d x} r_{2}(t)+\alpha_{2}(r(t))\right) d t+\sigma_{2}(r(t)) d W(t), \\
r(0)=r_{0} .
\end{array}\right.
$$

By the above calculations, the coefficient $\alpha_{2}$ is equal to

$$
\alpha_{2}(t)(x)=a(t, t+x)=V(t+x)-V\left(T^{*}\right), \quad t \geq 0, x \in[0, \infty) .
$$

We see that for a fixed $t \geq 0$, the function $c(t, \cdot)$ is at most Hölder-continuous of order strictly less than $\frac{1}{2}$ and therefore, the HJMM equations (4.38) and (4.39) need to be solved in larger spaces than the weighted $W^{1,2}$ spaces, for instance, weighted $L^{p}$ spaces or fractional Sobolev spaces.

\subsection{The HJMM equations with real-world dynamics driven by Wiener processes}

Tappe [55] presented new results concerning the HJMM equations with real-world dynamics. He assumed that under the real-world probability measure $\mathbb{P}$, for every $T$, 
the forward rate process $(f(t, T))_{t \in[0, T]}$ satisfies the term structure equation

$$
d f(t, T)=\alpha(t, T) d t+\sigma(t, T) d W(t)+\text { jumps, } \quad t \in[0, T],
$$

where $W$ is a (possibly infinite-dimensional) Wiener process. We did not write the jumps in (4.40) in detail since we assume that the jump terms in our case are simply equal to zero. If the jumps are zero, then Tappe's model includes the original HJMM framework. As argued in [37], switching to the Musiela parametrization [43], i.e.,

$$
r(t)(x)=f(t, t+x), \quad x \geq 0,
$$

leads to the partial differential equation

$$
\left\{\begin{array}{l}
d r(t)=\left(\frac{d}{d x} r(t)+\alpha(r(t))\right) d t+\sigma(r(t)) d W(t)+\text { jumps } \\
r(0)=r_{0}
\end{array}\right.
$$

Tappe proved that in an arbitrage-free bond market, the drift term in (4.41) is given by

$$
\alpha(t, T)=\sigma(t, T)\left(\theta(t)+\int_{t}^{T} \sigma(t, u) d u\right)+\text { jumps, } \quad t \in[0, T],
$$

where $\theta$ denotes the reference market price of risk with respect to the Wiener process $W$. He also proved, under some suitable conditions, the existence and uniqueness of mild solutions to the HJMM (4.41) with real-world dynamics in a separable Hilbert space.

Remark 4.28 We assume that the jumps are equal to zero. Let $W$ be an $H$-valued $\mathbb{F}$-cylindrical canonical Wiener process. Assume that $g:[0, \infty) \times \mathbb{R} \rightarrow \mathbb{R}$ is a given function and

$$
\sigma(r(t))(x)=g(x, r(t)(x)), \quad x \in[0, \infty), t \geq 0 .
$$

Then (4.41) becomes

$$
\left\{\begin{aligned}
d r(t)(x)= & \left(\frac{d}{d x} r(t)(x)+\langle g(x, r(t)(x)), \Psi(t)(x)\rangle_{H}\right) d t \\
& +\langle g(x, r(t)(x)), d W(t)\rangle_{H} \\
r(0)=r_{0}, &
\end{aligned}\right.
$$

where $\Psi(t)(x):=\theta(t)+\int_{0}^{x} g(y, r(t)(y)) d y, x \in[0, \infty), t \geq 0$.

Theorem 4.29 Assume $g:[0, \infty) \times \mathbb{R} \rightarrow H$ is a measurable function with respect to the first variable such that there exist functions $\bar{g} \in L_{v}^{p}$ and $\hat{g} \in L_{v}^{p} \cap L^{\infty}$ such that

$$
|g(x, u)| \leq|\bar{g}(x)|, \quad u \in \mathbb{R}, x \in[0, \infty)
$$


and

$$
|g(x, u)-g(x, v)| \leq|\hat{g}(x)||u-v|, \quad u, v \in \mathbb{R}, x \in[0, \infty) .
$$

Moreover, we assume that $\theta:[0, \infty) \times L_{\nu}^{p} \times \Omega \rightarrow H$ is a progressively measurable function such that there exist constants $C_{1}, C_{2}>0$ such that for all $t \geq 0$, we have

$$
\begin{array}{rlrl}
|\theta(t, f)| & \leq C_{1}\left(1+\|f\|_{\nu, p}\right), & & f \in L_{v}^{p}, \\
\left|\theta\left(t, f_{1}\right)-\theta\left(t, f_{1}\right)\right| \leq C_{2}\left\|f_{1}-f_{2}\right\|_{\nu, p}, & & f_{1}, f_{2} \in L_{v}^{p} .
\end{array}
$$

Then for each $r_{0} \in L^{2}\left(\Omega, \mathcal{F}_{0}, \mathbb{P} ; L_{v}^{p}\right),(4.42)$ has a unique $L_{v}^{p}$-valued continuous mild solution.

Acknowledgements The authors would like to thank Ben Goldys for a thorough discussion on the whole paper. They would also like to thank Ben Goldys, Enrico Priola and Jan van Neerven for a discussion related to Remark 3.15 and Mark Veraar for a discussion related to Remark 2.2. Finally, they would like to thank Guarv Dhariwal for a careful reading of the whole manuscript.

Open Access This article is distributed under the terms of the Creative Commons Attribution 4.0 International License (http://creativecommons.org/licenses/by/4.0/), which permits unrestricted use, distribution, and reproduction in any medium, provided you give appropriate credit to the original author(s) and the source, provide a link to the Creative Commons license, and indicate if changes were made.

\section{References}

1. Baudoin, F., Teichmann, J.: Hypoellipticity in infinite dimensions and an application in interest rate theory. Ann. Appl. Probab. 15, 1765-1777 (2005)

2. Baxendale, P.: Gaussian measures on function spaces. Am. J. Math. 98, 891-952 (1976)

3. Björk, T.: A Geometric View of the Term Structure of Interest Rates, Lecture notes written by Irene Crimaldi, Scuola Normale Superiore di Pisa, Classe di Scienze, Pisa, Cattedra Galileiana (2000)

4. Bogachev, V.I.: Measure Theory, vol. II. Springer, Berlin (2007)

5. Brzeźniak, Z.: Stochastic partial differential equations in M-type 2 spaces. Potential Anal. 4, 1-45 (1995)

6. Brzeźniak, Z.: On stochastic convolution in Banach spaces and applications. Stoch. Stoch. Rep. 61, 245-295 (1997)

7. Brzeźniak, Z., Carroll, A.: Approximation of the Wong-Zakai type for stochastic differential equations in $M$-type 2 Banach spaces with applications to loop spaces. In: Azéma, J., et al. (eds.) Séminaire de Probabilités XXXVII. Lecture Notes in Mathematics, vol. 1832, pp. 251-289. Springer, Berlin (2003)

8. Brzeźniak, Z., Elworthy, K.D.: Stochastic differential equations on Banach manifolds. Methods Funct. Anal. Topol. 6, 43-84 (2000)

9. Brzeźniak, Z., Ferrario, B.: Stationary solutions for stochastic damped Navier-Stokes equations in $\mathbb{R}^{d}$. To appear in Indiana Univ. Math. J., Available online at https://arxiv.org/abs/1702.00697, https:// www.iumj.indiana.edu/IUMJ/Preprints/7551.pdf

10. Brzeźniak, Z., Long, H., Simão, I.: Invariant measures for stochastic evolution equations in M-type 2 Banach spaces. J. Evol. Equ. 10, 785-810 (2010)

11. Brzeźniak, Z., Maslowski, B., Seidler, J.: Stochastic non-linear beam equations. Probab. Theory Relat. Fields 132, 119-149 (2005)

12. Brzeźniak, Z., Peszat, S.: Space time continuous solutions to SPDEs driven by a homogeneous Wiener process. Stud. Math. 137, 261-299 (1999)

13. Brzeźniak, Z., Peszat, S.: Stochastic two dimensional Euler equations. Ann. Probab. 29, 1796-1832 (2001) 
14. Brzeźniak, Z., Peszat, S.: Maximal inequalities and exponential estimates for stochastic convolutions in Banach spaces. In: Gesztesy, F., et al. (eds.) Stochastic Processes, Physics and Geometry: New Interplays, I: A Volume in Honor of Sergio Albeverio. CMS Conf. Proc., vol. 28. Am. Math. Soc., Providence (2000)

15. Brzeźniak, Z., Peszat, S., Zabczyk, J.: Continuity of stochastic convolutions. Czechoslov. Math. J. 51, 679-684 (2001)

16. Brzeźniak, Z., Veraar, M.: Is the stochastic parabolicity condition dependent on p and q? Electron. J. Probab. 17(56), 1-24 (2012)

17. Carroll, A.: The Stochastic Nonlinear Heat Equation, PhD Thesis, University of Hull (1999). Available online at https://ethos.bl.uk/OrderDetails.do;jsessionid=B60F597F18D1385C375EB9CB910543B6? uin=uk.bl.ethos.310216

18. Chojnowska-Michalik, A., Goldys, B.: Existence, uniqueness and invariant measures for stochastic semilinear equations on Hilbert spaces. Probab. Theory Relat. Fields 102, 331-356 (1995)

19. Da Prato, G.: An introduction to Markov semigroups. In: Iannelli, M., et al. (eds.) Functional Analytic Methods for Evolution Equations, CIRM Autumn School on Evolution Equations and Semigroups. Lecture Notes in Math., vol. 1855, pp. 1-63. Springer, Berlin (2004)

20. Da Prato, G., Röckner, M.: Dissipative stochastic equations in Hilbert space with time dependent coefficients. Atti Accad. Naz. Lincei, Rend. Lincei, Mat. Appl. 17, 397-403 (2006)

21. Da Prato, G., Röckner, M.: A note on evolution systems of measures for time-dependent stochastic differential equations. In: Dalang, R.C., et al. (eds.) Seminar on Stochastic Analysis, Random Fields and Applications V. Progr. Probab., vol. 59, pp. 115-122. Birkhäuser, Basel (2008)

22. Da Prato, G., Zabczyk, J.: Ergodicity for Infinite Dimensional Systems. Cambridge University Press, Cambridge (1996)

23. Dettweiler, E.: Banach spaces valued processes with independent increments and stochastic integration. In: Beck, A., Jacobs, K. (eds.) Proceedings of the Seminar Held in Oberwolfach, Germany, July 1982. Probability in Banach Spaces IV, pp. 54-83. Springer, Berlin (1983)

24. Sarhir, A.E., Farkas, B.: Invariant measure and regularity properties of perturbed Ornstein-Uhlenbeck semigroups. J. Differ. Equ. 233, 87-104 (2007)

25. Es Sarhir, A., Stannat, W.: Invariant measure for semilinear SPDEs with local Lipschitz drift coefficients and applications. J. Evol. Equ. 8, 129-154 (2008)

26. Filipović, D.: Consistency Problems of Heath-Jarrow-Morton Interest Rate Models. Springer, Berlin (2001)

27. Filipović, D., Teichmann, J.: On the geometry of the term structure of interest rates. Proc. R. Soc., Math. Phys. Eng. Sci. 460, 129-167 (2004)

28. Filipović, D., Tappe, S., Teichmann, J.: Term structure models driven by Wiener processes and Poisson measures: existence and positivity. SIAM J. Financ. Math. 1, 523-554 (2010)

29. van Gaans, O., Rusinek, A.: Invariant measures for stochastic evolution equations with the shift semigroup. Preprint (2010). Available online at http://www.math.leidenuniv.nl/reports/files/2010-10.pdf

30. Goldys, B., Musiela, M., Sondermann, D.: Lognormality of rates and term structure models. Stoch. Anal. Appl. 18, 375-396 (2000)

31. Goldys, B., Musiela, M.: Infinite dimensional diffusions, Kolmogorov equations and interest rate models. In: Jouini, E., et al. (eds.) Option Pricing, Interest Rates and Risk Management. Handbooks in Mathematical Finance, pp. 314-335. Cambridge University Press, Cambridge (2001)

32. Hairer, M., Labbé, C.: The reconstruction theorem in Besov spaces. Working paper (2017). Available online at https://arxiv.org/abs/1609.04543

33. Hairer, M., Mattingly, J.: A theory of hypoellipticity and unique ergodicity for semilinear stochastic PDEs. Electron. J. Probab. 16(23), 658-738 (2011)

34. Heath, D., Jarrow, R., Morton, A.: Bond pricing and the term structure of interest rates: a new methodology. Econometrica 61, 77-105 (1992)

35. Karatzas, L., Shreve, S.E.: Brownian Motion and Stochastic Calculus, 2nd edn. Springer, New York (1988)

36. Klein, I., Schmidt, T., Teichmann, J.: When roll-overs do not qualify as numéraire: bond markets beyond short rate paradigms. Working paper (2013). Available online at https://arxiv.org/abs/1310.0032

37. Kok, T.: Stochastic Evolution Equations in Banach Spaces and Application to Heath-Jarrow-MortonMusiela Equations, PhD Thesis, University of York, York (2017). Available online at http://etheses. whiterose.ac.uk/18070/

38. Kühn, T.: $\gamma$-Radonifying operators and entropy ideals. Math. Nachr. 107, 53-58 (1982)

39. Linde, W., Pietsch, A.: Mappings of Gaussian cylindrical measures in Banach spaces. Theory Probab. Appl. 19, 445-460 (1975) 
40. Liu, C., Prömel, D.J., Teichmann, J.: Stochastic analysis with modelled distributions. Working paper (2018). Available online at https://arxiv.org/abs/1609.03834

41. Marinelli, C.: Well-posedness and invariant measures for HJM models with deterministic volatility and Lévy noise. Quant. Finance 10, 39-47 (2010)

42. Metafune, G., Pallara, D., Priola, E.: Spectrum of Ornstein-Uhlenbeck operators in $L^{p}$ spaces with respect to invariant measures. J. Funct. Anal. 196, 40-60 (2002)

43. Musiela, M.: Stochastic PDEs and term structure models, J. Intern. Finance, IGR-AFFI, La Baule (1993). Unpublished

44. Neidhardt, A.L.: Stochastic Integrals in 2-Uniformly Smooth Banach Spaces, PhD Thesis, University of Wisconsin (1978). Unpublished

45. Van Neerven: $y$-Radonifying operators-a survey. In: Hassel, A., et al. (eds.) Proc. Centre Math. Appl., the AMSI-ANU Workshop on Spectral Theory and Harmonic Analysis, pp. 1-61. Austral. Nat. Univ., Canberra (2010)

46. van Neerven, J., Veraar, M., Weis, L.: Stochastic maximal $L^{p}$-regularity. Ann. Probab. 40, 788-812 (2012)

47. Ondreját, M.: Brownian representations of cylindrical local martingales, martingale problem and strong Markov property of weak solutions of SPDEs in Banach spaces. Czechoslov. Math. J. 55, 1003-1039 (2005)

48. Ouyang, S., Röckner, M.: Time inhomogeneous generalized Mehler semigroups and skew convolution equations. Forum Math. 28, 339-376 (2016)

49. Pazy, A.: Semigroups of Linear Operators and Applications to Partial Differential Equations. Springer, New York (1983)

50. Peszat, S., Zabczyk, J.: Stochastic Partial Differential Equations with Lévy Noise. Cambridge University Press, Cambridge (2007)

51. Pisier, G.: Martingales with values in uniformly convex spaces. Isr. J. Math. 20, 326-350 (1975)

52. Stroock, D., Varadhan, S.: Multidimensional Diffusion Processes. Reprint of the 1997 edition. Classics in Mathematics. Springer, Berlin (2006)

53. Vargiolu, T.: Invariant measures for the Musiela equation with deterministic diffusion term. Finance Stoch. 3, 483-492 (1999)

54. Tehranchi, M.: A note on invariant measures for HJMM models. Finance Stoch. 9, 389-398 (2005)

55. Tappe, S.: The HJMM equation with real-world dynamics driven by Wiener processes and Poisson random measures. Working paper (2012). Available online at https://www.stochastik.uni-hannover. de/fileadmin/institut/pdf/Ex-Pos.pdf

56. Yosida, K.: Functional Analysis, 6th edn. Springer, Berlin (1980)

57. Zhu, J., Brzeźniak, Z., Hausenblas, E.: Maximal inequalities for stochastic convolutions driven by compensated Poisson random measure in Banach spaces. Ann. Inst. Henri Poincaré B, Probab. Stat. 53, 937-956 (2015) 